\title{
NATURAL MODES OF BERNOULLI-EULER BEAMS WITH A SINGLE-EDGE CRACK
}

\author{
Mo-How H. Shen* \\ Department of Aeronautical and Astronautical Engineering \\ The Ohio State University, Columbus, Ohio \\ Christophe Pierre* \\ Department of Mechanical Engineering and Applied Mechanics \\ The University of Michigan, Ann Arbor, Michigan
}

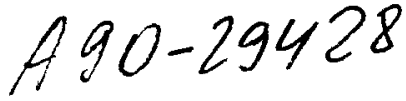

\begin{abstract}
$\underline{\text { Abstract }}$
The equation of motion and associated boundary conditions are derived for a uniform Bernoulli-Euler beam containing one single-edge crack. The main idea is to use a generalized variational principle that allows for modified stress, strain, and displacement fields that enable one to satisfy the compatibility requirements in the vicinity of the crack. The concentration in stress is represented by introducing a crack function into the beam's compatibility relations. A displacement function is also introduced to modify the in-plane displacement and its slope near the crack. Both functions are chosen to have their maximum value at the cracked section and to decay exponentially along the beam's longitudinal direction. The rate of exponential decay is evaluated from finite element calculations. The resulting equation of motion is solved for simply supported and cantilevered beams with single-edge cracks by a Galerkin and a local Ritz procedure, respectively. These theoretical natural frequencies and mode shapes are confirmed by comparisons with experimental and finite element results, and in both cases a close match is obtained. The possibility of determining the cracked beams' damage properties from the changes of its dynamic behavior is discussed.
\end{abstract}

\section{Nomenclature}

$\begin{array}{ll}a & \text { crack depth } \\ a_{i} & \text { ith generalized coordinate amplitude } \\ A & \text { beam cross section area } \\ b & \text { half breadth of rectangular beam } \\ C R & \equiv \frac{a}{2 d}, \text { crack ratio } \\ d & \text { half depth of rectangular beam } \\ E & \text { Young's modulus of elasticity } \\ f(x, z) & \text { crack function } \\ F R & \equiv \frac{\omega_{c}}{\omega_{u c}}, \text { frequency ratio } \\ H(.) & \text { unit step function } \\ I & \text { cross-sectional area moment of interia } \\ {\left[k_{i}\right]} & \text { element stiffness matrix } \\ {\left[K_{e}\right]} & \text { global stiffness matrix } \\ K & \equiv \int_{A} z f d A \\ K_{1} & \equiv \int_{A} z \varphi d A \\ K_{2} & \equiv \int_{A} z \varphi^{\prime} d A \\ \bar{K}_{1} & \equiv K_{1} / I \\ \bar{K}_{2} & \equiv K_{2} / I \\ l & \text { length of beam }\end{array}$

* Assistant Professor. Member AIAA, ASME

Copyright (C) 1990 by Mo-How H. Shen. Published by the American Institute of Aeronautics and Astronautics, Inc. with permission.

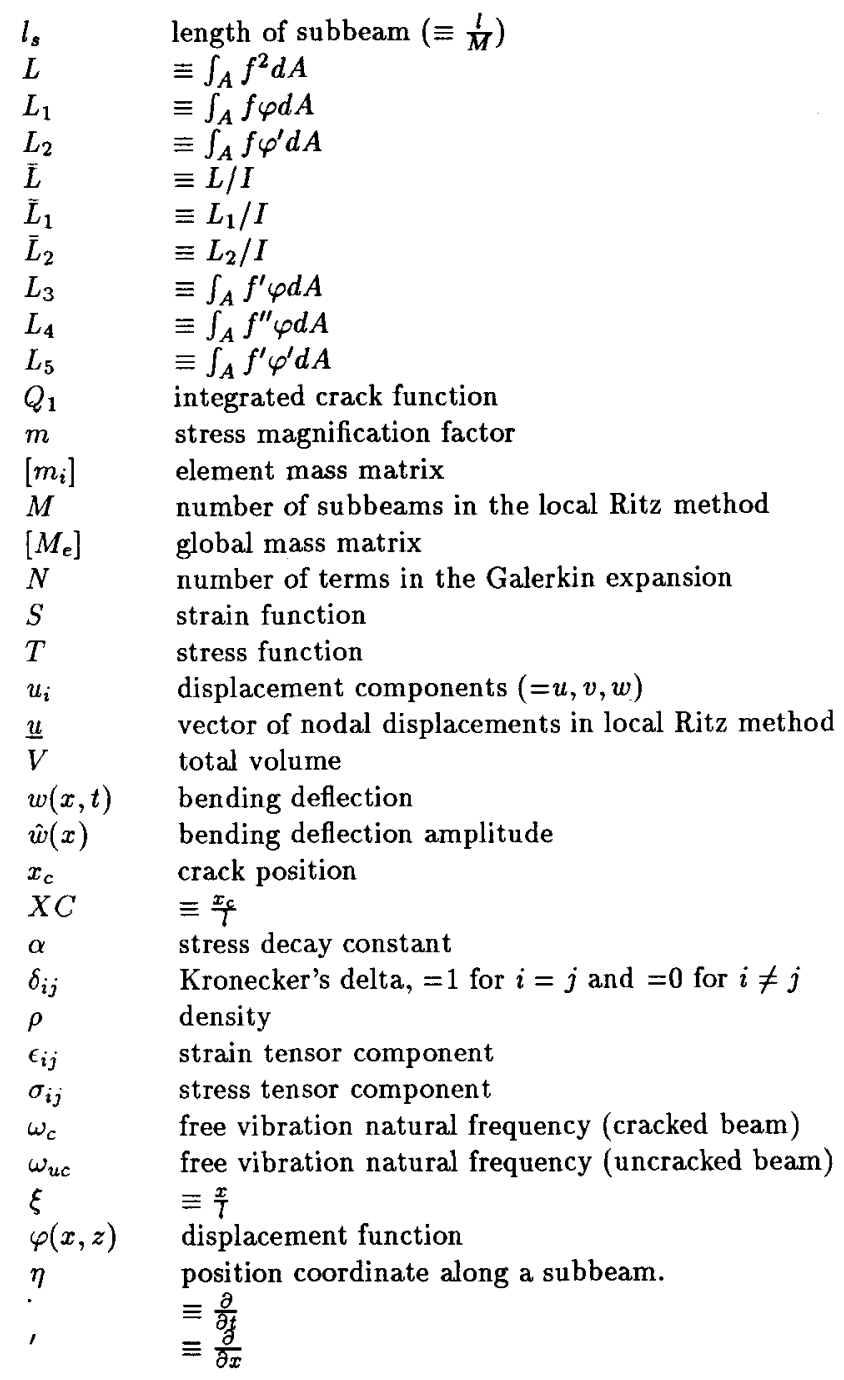

\section{Introduction}

The development of damage identification techniques for vibrating structures such as turbines, generators, motors, aircraft structures, and large space structures has recently become the focus of substantially growing research efforts. Due to in- 
creasing demands for safety, reliability, and time-efficiency, it is now believed that the monitoring of the global dynamics of the structure offers promising alternatives for damage detection. Consequently, the study of the dynamics of cracked structures is of importance.

Relatively few investigators have examined the dynamics of cracked structures. For example, the effects of cracks on the dynamic behavior of beams was studied by Chondros and Dimarogonas [1], Dimarogonas and Massouros [2], and Dimarogonas and Papadopoulos [3]. They modeled the crack by introducing a local flexibility matrix connecting longitudinal, bending, and shear forces and displacements. Later, Gudmundson [4] and several other researchers generalized this idea to a $6 \times 6$ flexibility matrix relating all six generalized forces to the corresponding displacements, and applied it to a variety of dynamic problems. Torsion was also considered by Papadopoulos and Dimarogonas [5], who derived a more complicated flexibility matrix. They showed that a crack in a Timoshenko shaft introduces couping between torsion and shear. Since in the Timoshenko beam model there is coupling between the shear and bending deformations, the torsional motion is coupled to the bending one for a cracked shaft.

Cawley and Adams $[6,7]$ demonstrated the feasibility of using natural frequency test measurements to detect damage in a structure. Their approach consisted in comparing the natural frequencies obtained from finite element analysis with the measured frequencies. They introduced damage in the finite element model by a reduced stiffness element. The damage location was determined by replacing each element with a reduced stiffness element until the finite element frequencies matched best the measured frequencies.

Recently, Christides and Barr [8] derived the equation of bending motion for a Bernoulli-Euler beam containing pairs of symmetric cracks. The cracks were taken to be normal to the beam's neutral axis and symmetrical about the plane of bending. They used an exponential-type function (the so-called "crack function") to model the stress concentration near the crack tip. The rate of stress decay from the crack was controlled by a dimensionless parameter, $\alpha$, that was determined by fitting the analytical results to experimental data. However, Christides and Barr obtained the approximate cracked beam natural frequencies by a two-term Rayleigh Ritz procedure. Recently, Shen and Pierre [9] showed that this two-term solution does not feature adequate convergence and that, indeed, convergence is very slow for this type of problems, because cracks affect the continuity characteristics of the solution. To insure adequate convergence, an approximate Galerkin solution with as many as 150 terms was suggested in [9], which led to a redetermination of the stress decay rate $\alpha$. To validate the theoretical results, a two-dimensional finite element approach was also proposed in [9], which allows one to determine $\alpha$ without requiring the use of experimental results.

The cracked beam theory in Refs. $[8,9]$ is restricted to pairs of symmetric cracks. This assumption was made to avoid the modeling difficulty due to the discontinuities in the slope of the neutral axis and in the axial displacement along the neutral axis, which both occur with a non-symmetric crack configuration. The crack beam theory of Refs. [8] and [9] is further extended in the present study, which investigates the effects of single surface cracks on the modes of free vibration of beams. The analysis proceeds in several steps. First, we assume that the damage at a particular cross section can be viewed as a single surface crack, which is taken to be normal to the beam's neutral axis. According to the observations of Freund [10-12], Bodner [13], and Freund and Herrmann [14] that the normal stress distribution on the prospective fracture plane is essentially linear before initiation of the fracture on the tensile side of the beam, a crack function $f$ is introduced into the normal stress and strain expressions to account for this phenomenon. Also, a function is introduced in the representation of the inplane displacement to model the disruption of the deformation field due to the crack. A generalized variational principle extended from the $\mathrm{Hu}$-Washizu principle is used to develop the governing equations for a uniform beam with a single-edge crack. This procedure is similar to that used for the flexural vibration of beams with pairs of symmetric cracks $[8,9]$. These equations and boundary conditions are particularized for a cracked beam with a uniform rectangular cross section. The Galerkin and Ritz methods are then applied to predict the free vibration modes of cracked beams, for both simply supported and cantilevered configurations. The value of the stress decay factor $\alpha$ is determined by a least square fit of the natural frequencies calculated by Galerkin or Ritz methods with finite element results. The value of $\alpha$ is found to be 1.979 for simply supported beams and 1.930 for the cantilevered beam - close to the values found in Ref. [9].

Two basic issues are addressed in this study. First, the adequacy of a model based on a simple beam theory for the prediction of the dynamic response of cracked beams is demonstrated. Second, the effects of a single surface crack on the free response of simply supported and cantilevered beams are investigated. The possible use of this formulation to identify the crack position and size from changes in the beams' natural frequencies and mode shapes is also discussed.

\section{Cracked Beam Theory for Single-Edge}

\section{Cracks}

\section{Kinematic assumptions}

The distribution of stress and strain in an elastic body with a crack has been studied by Irwin [15] and Paris and Sih [16]. They divided the stress fields near the crack-tip into three basic types, each associated with a local mode of deformation. These are mode I, the crack-edge opening mode; mode II, the crackedge sliding mode; and mode III, the crack-edge tearing mode.

In the case of free bending vibrations of a uniform beam with a single-edge crack, the bending moment and the longitudinal force do not contribute to mode II and mode III deformations. The shear force does contribute to mode II deformation. However, for slender beams, this contribution can be neglected.

Similar to the case of symmetric cracks, the normal stress, $\sigma_{x x}$, is the only stress that is affected by a single crack. The remaining normal and shear stresses out of the plane of bending are assumed to be zero. The in-plane shear stress component, $\sigma_{x z}$, is included in order to accommodate the possibility of shear loading on the lateral surfaces of the beam. Since $\sigma_{x z}$ is not concentrated in mode $I$, the details of its distribution are not affected by the crack.

In Refs. [15] and [16], the stress component $\sigma_{x x}$ was found to be concentrated at the crack-tip and to decay in inverse proportion to the square root of the distance from the cracktip. This phenomenon is reproduced here by using a crack function $f(x, z)$ in the expressions of the stress $\sigma_{x x}$ and the strain $\epsilon_{x x}$, as follows:

$$
\begin{aligned}
& \sigma_{x x}(x, z, t)=(-z+f(x, z)) T(x, t) \\
& \epsilon_{x x}(x, z, t)=(-z+f(x, z)) S(x, t)
\end{aligned}
$$

where $T(x, t)$ and $S(x, t)$ are defined as unknown stress and 
strain functions, respectively.

The function $f(x, z)$ has its maximum value at the cracktip. It is taken to decay exponentially along the length of the beam and to vary linearly through the depth of the uncracked portion of the beam, according to:

$$
f(x, z)=\left[z-m\left(z+\frac{a}{2}\right) H((d-a)-z)\right] \exp \left[-\alpha \frac{\left|x-x_{c}\right|}{d}\right]
$$

Here $x_{c}, a$, and $d$ represent the crack position, the crack length, and half the depth of the uncracked section, respectively, as shown in Fig. 1. The positive nondimensional constant $\alpha$ determines the rate of stress decay away from the crack tip. It was determined to be 1.936 in Ref. [9] for a pair of symmetric cracks. At the crack section $x_{c}$ and for $z>(d-a)$ (i.e., within the crack $)$, the unit step function $H((d-a)-z)$ has value zero so that $f(x, z)$ reduces to $z$ and the stress and strain in Eqs. (1, 2 ) have value zero. The constant $m$ represents the slope of the linear stress distribution at the cracked section. It can be estimated by applying the condition that the same bending moment is carried by both cracked and uncracked beams at the crack-tip section.

The axial displacement $u(x, z, t)$ is represented in terms of its derivative $u^{\prime}$ as

$$
u^{\prime}(x, z, t)=(-z+\varphi(x, z)) w^{\prime \prime}(x, t)
$$

where $w(x, t)$ is the transverse beam deflection. The function $\varphi$ is chosen so that the surface of zero in-plane displacement and its slope coincide with the surface of zero normal stress and normal strain, respectively. For (kinematic) consistency between Eqs. (2) and (4) we choose $\varphi(x, z)$ to be similar in form to $f(x, z)$ in $\mathrm{Eq} .(3)$ :

$$
\varphi(x, z)=\left[z-\left(z+\frac{a}{2}\right) H((d-a)-z)\right] \exp \left(-\alpha \frac{\left|x-x_{c}\right|}{d}\right)
$$

The assumptions for a nominally uniform beam with a single surface crack are summarized as

$$
\left\{\begin{array}{l}
u_{y}=0, u_{z}=w(x, t) \\
p_{x}=0, p_{y}=0, p_{z}=P(x, t) \\
\epsilon_{x x}=(-z+f(x, z)) S(x, t) \\
\epsilon_{y y}=\epsilon_{z z}=-\nu \epsilon_{x x} \\
\epsilon_{x y}=\epsilon_{y z}=\epsilon_{x z}=0 \\
\sigma_{x x}=(-z+f(x, z)) T(x, t), \sigma_{x z}=\sigma_{x z}(x, z, t) \\
\sigma_{y y}=\sigma_{z z}=\sigma_{x y}=\sigma_{y z}=0 \\
X_{x}=X_{y}=X_{z}=0
\end{array}\right.
$$

where the $u_{i}$ are the displacements referring to cartesian axes $x, y, z ; \sigma_{i j}$ and $\epsilon_{i j}$ represent stress and strain; and $X_{i}$ and $p_{i}$ are the body forces and velocity components, respectively. The shear stress $\sigma_{x z}$ is included to permit the loading of the beam.

\section{Variational theorem}

Since $S, T, P$, and $w$ are unknown functions, the compatibility and constitutive relations of the cracked beam are undefined. In the absence of these relations, classical variational principles such as Hamilton's principle are inadequate. However, these principles can be generalized by the introduction of Lagrange multipliers to yield a family of variational principles that includes the Hellinger-Reissner principle in elastodynamic problems and the Hu-Washizu principle in elastic static problems.

Here, the $\mathrm{Hu}$-Washizu principle is modified to include the virtual work done by the inertial forces. This yields the following functional:

$$
J=\int_{t_{1}}^{t_{2}}\left\{\int _ { V } \left[\rho p_{i} \dot{u}_{i}-\frac{1}{2} \rho p_{i} p_{i}-\underline{A}\left(\epsilon_{i j}\right)+\left(\epsilon_{i j}-\frac{1}{2}\left(u_{i, j}+u_{j, i}\right)\right) \sigma_{i j}\right.\right.
$$

$$
\left.\left.+X_{i} u_{i}\right] d V+\int_{S_{1}} \bar{g}_{i} u_{i} d S_{1}+\int_{S_{2}} g_{i}\left(u_{i}-\bar{u}_{i}\right) d S_{2}\right\} d t
$$

where $\rho$ is the density, $\underline{A}\left(\epsilon_{i j}\right)$ is the strain energy density function, the $g_{i}^{\prime} s$ are the surface tractions, $V$ is the total volume of the system, and $S$ is its external surface. The overbarred quantities $\bar{g}_{i}$ and $\bar{u}_{i}$ denote the prescribed values of surface tractions and surface displacements, respectively.

The functional $J$ in Eq. (7) is stationary for the actual solution in the independent quantities $u_{i}, p_{i}, \epsilon_{i j}$, and $\sigma_{i j}$. Therefore, for arbitrary independent variations of $\delta u_{i}$ (with conditions $\left.\delta u\left(t_{1}\right)=\delta u\left(t_{2}\right)=0\right), \delta p_{i}, \delta \epsilon_{i}$, and $\delta \sigma_{i}$, the first variation of $J$ must be equal to zero, yielding

$$
\begin{gathered}
\delta J=\int_{t_{1}}^{t_{2}}\left\{\int _ { V } \left\{\left(\sigma_{i j, j}+X_{i}-\rho \dot{p}_{i}\right) \delta u_{i}+\left(\sigma_{i j}-\underline{A}_{, \epsilon_{i j}}\right) \delta \epsilon_{i j}\right.\right. \\
\left.+\left[\epsilon_{i j}-\frac{1}{2}\left(u_{i, j}+u_{j, i}\right)\right] \delta \sigma_{i j}+\left[\rho \dot{u}_{i}-\left(\frac{1}{2} \rho p_{i} p_{i}\right)_{, p_{i}}\right] \delta p_{i}\right\} d V \\
\left.+\int_{S_{1}}\left(\bar{g}_{i}-g_{i}\right) \delta u_{i} d S_{1}+\int_{S_{2}}\left(u_{i}-\bar{u}_{i}\right) \delta g_{i} d S_{2}\right\} d t=0
\end{gathered}
$$

\section{Equation of motion and associated boundary}

\section{conditions}

The assumptions (6) are substituted into the formulation (8), whereby the problem is reduced to a form corresponding to the beam model. After integration by parts and simplification, we obtain the following:

\section{Strain-displacement term}

The strain-displacement term in Eq. (8) is given by

$$
\begin{gathered}
\int_{V}\left(\epsilon_{x x}-\frac{\partial u}{\partial x}\right) \delta \sigma_{x x} d V= \\
\int_{x}\left\{\int_{A}\left[(f-z) S-(\varphi-z) w^{\prime \prime}\right](f-z) \delta T d A\right\} d x
\end{gathered}
$$

Using the following definitions,

$$
\begin{gathered}
I=\int_{A} z^{2} d A, \quad K=\int_{A} z f d A, \quad L=\int_{A} f^{2} d A, \\
K_{1}=\int_{A} z \varphi d A, \quad L_{1}=\int_{A} f \varphi d A
\end{gathered}
$$

the right-hand side of Eq. (9) can be rewritten as

$$
\int_{x}\left[(I-2 K+L) S-\left(I+L_{1}-K-K_{1}\right) w^{\prime \prime}\right] \delta T d x
$$

\section{Strain-stress term}

The strain-stress term in Eq. (8) is given as

$$
\int_{V}\left[\left(\sigma_{x x}-\frac{\partial \underline{A}}{\partial \epsilon_{x x}}\right) \delta \epsilon_{x x}-\frac{\partial \underline{A}}{\partial \epsilon_{y y}} \delta \epsilon_{y y}-\frac{\partial \underline{A}}{\partial \epsilon_{z z}} \delta \epsilon_{z z}\right] d V
$$

If the material is elastic and isotropic, we have

$$
\frac{\partial \underline{A}}{\partial \epsilon_{x x}}=E \epsilon_{x x}, \frac{\partial \underline{A}}{\partial \epsilon_{y y}}=0, \frac{\partial \underline{A}}{\partial \epsilon_{z z}}=0,
$$

so Eq. (12) becomes

$$
\int_{V}\left(\sigma_{x x}-E \epsilon_{x x}\right) \delta \epsilon_{x x} d V=\int_{x}(T-E S)(I-2 K+L) \delta S d x
$$

\section{Velocity term}

The velocity term in Eq. (8) is 


$$
\int_{x}(\rho A \dot{w}-\rho P A) \delta P d x
$$

\section{Dynamic equilibrium term}

The first term in Eq. (8) represents the virtual work done by the dynamic forces. In the absence of body forces, it can be written as

$$
\int_{V}\left\{\frac{\partial \sigma_{x x}}{\partial x} \delta u+\frac{\partial \sigma_{x z}}{\partial z} \delta u+\left(\frac{\partial \sigma_{x z}}{\partial x}-\rho \dot{P}\right) \delta w\right\} d V
$$

Under the assumptions (6), the expression (16) becomes

$$
\begin{gathered}
\int_{V}\left\{\left([(f-z) T]^{\prime}+\right.\right. \\
\left.+\frac{\partial \sigma_{x z}}{\partial z}\right) \varphi \delta w^{\prime}-\left([(f-z) T]^{\prime}+\frac{\partial \sigma_{x z}}{\partial z}\right) z \delta w^{\prime} \\
\left.+\left(\frac{\partial \sigma_{x z}}{\partial x}-\rho \dot{P}\right) \delta w\right\} d V
\end{gathered}
$$

Using the definitions

$$
\begin{gathered}
K_{2}=\int_{A} z \varphi^{\prime} d A, \quad L_{2}=\int_{A} f \varphi^{\prime} d A, \quad L_{3}=\int_{A} f^{\prime} \varphi d A, \\
L_{4}=\int_{A} f^{\prime \prime} \varphi d A, \quad L_{5}=\int_{A} f^{\prime} \varphi^{\prime} d A
\end{gathered}
$$

and integrating by parts the first two terms in Eq. (17), we obtain

$$
\begin{aligned}
& \left.\int_{A}\left\{[(f-z) T]^{\prime}+\frac{\partial \sigma_{x z}}{\partial z}\right\} \varphi \delta w d A\right|_{\substack{x=0 \\
x=0}} ^{x=1}-\left.\int_{y} \int_{x} \frac{\partial\left(\varphi \sigma_{x z}\right)}{\partial x} \delta w d x d y\right|_{z=-d} ^{z=d} \\
& -\int_{x}\left\{\left(L_{1}-K_{1}\right) T^{\prime \prime}+2 T^{\prime} L_{3}+T L_{4}+\left(L_{2}-K_{2}\right) T^{\prime}+L_{5} T\right\} \delta w d x
\end{aligned}
$$

Integrating by parts the last two terms of Eq. (17) yields

$$
\begin{aligned}
& -\left.\int_{A}\left\{[(f-z) T]^{\prime}+\frac{\partial \sigma_{x z}}{\partial z}\right\} z \delta w d A\right|_{\substack{x=0 \\
x=1}}+\left.\int_{y} \int_{x} \frac{\partial\left(z \sigma_{x z}\right)}{\partial x} \delta w d x d y\right|_{z=-d} ^{z=d} \\
& -\int_{x} \int_{A} \frac{\partial \sigma_{x z}}{\partial x} \delta w d A d x+\int_{x}\left\{(K-I) T^{\prime \prime}+2 T^{\prime} K^{\prime}+T K\right\} \delta w d x(20)
\end{aligned}
$$

Finally, substituting Eqs. (19, 20) into Eq. (17) and integrating over the cross section $A$, we have

$$
\begin{aligned}
& \int_{x}\left\{\left[\left(K-I-L_{1}+K_{1}\right) T^{\prime \prime}+\left(2 K^{\prime}-2 L_{3}-L_{2}+K_{2}\right) T^{\prime}\right.\right. \\
& \left.\left.+\left(K^{\prime \prime}-L_{4}-L_{5}\right) T\right]-\rho A \dot{P}\right\} \delta w d x+\int_{A}\left\{[(f-z) T]^{\prime}(\varphi-z)\right.
\end{aligned}
$$

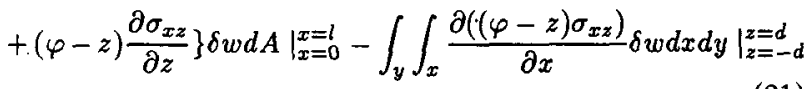

The last two terms in Eq. (8) represent the boundary conditions for the ends and the lateral surfaces of the beam. They are incorporated with the other boundary conditions as follows:

\section{Boundary force terms}

- Lateral surfaces: It is assumed that the lateral surfaces of the beam are free of external traction, i.e., $\bar{g}_{i}=0$ on these surfaces. This assumption comes from the relationship between $g_{i}$ and $\sigma_{i j}$ (given by Cauchy's formula)

$$
g_{i}=\nu_{j} \sigma_{i j}
$$

where $\nu_{i}$ is the unit outer normal vector. Since $\nu_{x}$ and $\nu_{y}$ are zero and $\nu_{z}$ is 1 on the lateral surface, Eq. (22) becomes:

$$
g_{x}=\sigma_{x z}
$$

$$
\begin{aligned}
& g_{y}=0 \\
& g_{z}=0
\end{aligned}
$$

Accordingly, the condition that the lateral surfaces are tractionfree corresponds to the requirement that $\sigma_{x z}=0$ on these surfaces.

The boundary force term in Eq. (8) over the lateral surface is

$$
\begin{gathered}
\int_{x} \int_{y}\left[\left.\left(0-\sigma_{x z}\right)\right|_{z=-d} \delta u+\left.\left(0+\sigma_{x z}\right)\right|_{z=d} \delta u\right] d x d y \\
=\left.\int_{x} \int_{y}\left(-\sigma_{x z}\right) \delta u d x d y\right|_{\substack{z=d \\
z=-d}} ^{z=d}
\end{gathered}
$$

Integrating by parts over $x$ yields

$$
\begin{gathered}
{\left[\left.\left(\int_{y}(\varphi-z)\left(-\sigma_{x z}\right) \delta w d y\right)\right|_{x=0} ^{x=1} x\right.} \\
\left.-\int_{x} \int_{y} \frac{\partial\left\{(\varphi-z)\left(-\sigma_{x z}\right)\right\}}{\partial x} \delta w d x d y\right]\left.\right|_{z=-d} ^{z=d}
\end{gathered}
$$

The second term in Eq. (25) cancels the last term in Eq. (21). The remaining force term in Eq. (21) can be integrated by parts over $z$ and results in a term that is cancelled by the first term in Eq. (25). The remaining term turns out to be the end condition:

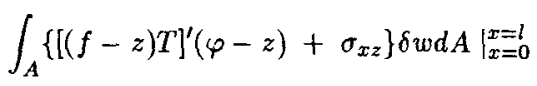

- End surfaces: In the same way, we may determine $\nu_{x}$ to be -1 and +1 over the ends of the beam, $x=0, l$, by assuming the plane ends to be normal to the beam axis. According to Cauchy's formula (22), $g_{x}$ reduces to $\pm \sigma_{x x}$ and $g_{z}$ to $\pm \sigma_{x z}$ at $x=0$ and $x=l$, respectively. The external tractions at the ends, $\bar{g}_{i}$, are prescribed as $\bar{X}$ and $\bar{Z}$. The force boundary term in Eq. (8) is therefore

$$
\begin{aligned}
& \left.\int_{A}\left\{\left(\bar{X}-\sigma_{x x}\right) \delta u+\left(\bar{Z}-\sigma_{x z}\right) \delta w\right\} d A\right|_{x=l} \\
+ & \left.\int_{A}\left\{\left(\bar{X}+\sigma_{x x}\right) \delta u+\left(\bar{Z}+\sigma_{x z}\right) \delta w\right\} d A\right|_{x=0}
\end{aligned}
$$

By incorporating Eq. (26) with Eq. (27) and substituting the relations (6), the final boundary force terms become:

$$
\begin{gathered}
{\left[\int_{A}\left\{(\varphi-z) \bar{X} d A-T\left(I+L_{1}-K-K_{1}\right)\right\} \delta w^{\prime}\right.} \\
\left.+\int_{A}\left\{\bar{Z} d A+T^{\prime}\left(I+L_{1}-K-K_{1}\right)+T\left(L_{3}-K^{\prime}\right)\right\} \delta w\right]\left.\right|_{x=l} \\
+\left[\int_{A}\left\{(\varphi-z) \bar{X} d A+T\left(I+L_{1}-K-K_{1}\right)\right\} \delta w^{\prime}\right. \\
\left.+\int_{A}\left\{\bar{Z} d A-T^{\prime}\left(I+L_{1}-K-K_{1}\right)+T\left(L_{3}-K^{\prime}\right)\right\} \delta w\right]\left.\right|_{x=0}
\end{gathered}
$$

\section{Boundary displacement terms}

With $\bar{u}$ and $\bar{w}$ as the prescribed displacements at the ends $x=0, l$, the boundary displacement terms in Eq. (8) are

$$
\begin{gathered}
\left.\int_{A}\left\{(u-\bar{u}) \delta \sigma_{x x}+(w-\bar{w}) \delta \sigma_{x z}\right\} d A\right|_{x=l} \\
-\left.\int_{A}\left\{(u-\bar{u}) \delta \sigma_{x x}+(w-\bar{w}) \delta \sigma_{x z}\right\} d A\right|_{x=0}
\end{gathered}
$$

Substituting for $u$ and $\sigma_{x x}$ from Eq. (6) and integrating over the cross section yields

$$
\left[\left\{\left(I+L_{1}-K-K_{1}\right) w^{\prime}-\int_{A} \bar{u}(f-z) d A\right\} \delta T+\left.\left[(w-\bar{w}) A \delta \sigma_{x z}\right]\right|_{x=l}-\right.
$$


$\left[\left\{\left(I+L_{1}-K-K_{1}\right) w^{\prime}-\int_{A} \bar{u}(f-z) d A\right\} \delta T+\left.\left[(w-\bar{w}) A \delta \sigma_{x z}\right]\right|_{x=0}\right.$

Derivation of equation of motion

Finally, the variational terms (11), (14), (15), and (21) are substituted into Eq. (8) along with the boundary terms $(28)$ and $(30)$. Since the variations $\delta w, \delta P, \delta S$, and $\delta T$ are independent, each quantity multiplied by the corresponding variation must equal zero. This leads to, from expression (11)

$$
S=Q_{1}(x) w^{\prime \prime}
$$

where

$$
Q_{1}=\frac{I+L_{1}-K-K_{1}}{I-2 K+L}
$$

The above equation shows that $(I-2 K+L)$ necessarily differs from zero. Therefore, from expression (14)

$$
T=E S
$$

From expression (15)

$$
P=\dot{w}
$$

From expression (21)

$$
\begin{gathered}
\left(K-I-L_{1}+K_{1}\right) T^{\prime \prime}+\left(2 K^{\prime}-2 L_{3}-L_{2}+K_{2}\right) T^{\prime} \\
+\left(K^{\prime \prime}-L_{4}-L_{5}\right) T-\rho A \dot{P}=0
\end{gathered}
$$

Equation (35) can be rewritten in terms of the displacement $w$ by substituting $S, T$, and $P$ from (31), (33), and (34). This leads to the equation of motion

$$
\begin{gathered}
E\left(I+L_{1}-K-K_{1}\right) Q_{1} w^{\prime \prime \prime \prime}+E\left[2\left(I+L_{1}-K-K_{1}\right) Q_{1}^{\prime}\right. \\
\left.+\left(2 L_{3}+L_{2}-K_{2}+2 K^{\prime}\right) Q_{1}\right] w^{\prime \prime \prime}+E\left[\left(I+L_{1}-K-K_{1}\right) Q_{1}^{\prime \prime}\right. \\
\left.+\left(2 L_{3}+L_{2}-K_{2}-2 K^{\prime}\right) Q_{1}^{\prime}+\left(L_{4}+L_{5}-K^{\prime \prime}\right) Q_{1}\right] w^{\prime \prime}+\rho A \ddot{w}=0
\end{gathered}
$$

\section{Derivation of boundary conditions}

The boundary conditions are of two kinds:

A. Specified displacements. The boundary conditions are obtained by equating the surface integral expression (28) to zero when the displacements $u$ and $w$ are prescribed on the boundary.

B. Specified forces. The boundary conditions are obtained by equating the surface integral expression (30) to zero when the external forces $T$ and $\sigma_{x z}$ are prescribed on the boundary.

For example, let us consider a cantilevered beam with a fixed end at $x=0$. The virtual displacements $\delta u$ (i.e., $\delta w^{\prime}$ ) and $\delta w$ must vanish at $x=0$. From Eq. (28), this implies that the virtual forces $\delta T$ and $\delta \sigma_{x z}$ are arbitrary. With zero displacements, $\vec{u}$ and $\tilde{w}$, at $x=0$, Eq. (30) gives

$$
w=0, w^{\prime}=0
$$

At $x=l$, the external forces $\bar{X}$ and $\bar{Z}$ are zero, and Eq. (28) gives

$$
T\left(I+L_{1}-K-K_{1}\right)=0
$$

and

$$
T^{\prime}\left(I+L_{1}-K-K_{1}\right)+T\left(L_{3}-K^{\prime}\right)=0
$$

Since $\left(I+L_{1}-K-K_{1}\right)$ differs from zero, the above two equations imply that $w^{\prime \prime}=0$ (from $T=0$ ) and that $w^{\prime \prime \prime}=0$ (from $\left.T^{\prime}=0\right)$.
Clearly, if there is no crack, the functions $L, L_{1}, K$ and $K_{1}$ are zero, and $Q_{1}$ becomes unity. The equation of motion and associated boundary conditions are then reduced to those of the uniform uncracked Bernoulli-Euler beam.

As pointed out earlier, the above cracked beam theory is based on the stress and strain distributions, Eqs. $(1,2)$, and the slope of the in-plane displacement, Eq. (4). The stress (or strain) distribution is characterized by the crack function (3), with the parameters $\alpha$ and $m$ defining the stress profiles in the $x$ and $z$ directions. The parameter $\alpha$ is evaluated in section 3 , in a least square sense. Since the stress along the $z$ axis is assumed to be linear, its decay rate $m$ can be estimated from the condition that the same bending moment is carried by the cracked beam the uncracked beam at the crack section:

$$
\int_{A}\left(-z E w^{\prime \prime}\right) z d A=\int_{A_{c}}\left(-z+f\left(x_{c}, z\right)\right) E S\left(x_{c}, t\right)\left(z+\frac{a}{2}\right) d A(40)
$$

where $A_{c}$ represents the cross sectional area at the crack-tip $\left(x=x_{c}\right)$, and the left-hand side of Eq. (40) is for the uncracked beam.

At the crack-tip section, we have, from Eqs. (3), (31) and $(32)$

$$
\begin{gathered}
f\left(x_{c}, z\right)=z-m\left(z+\frac{a}{2}\right) \\
Q_{1}\left(x_{c}\right)=\frac{1}{m} \\
S\left(x_{c}, t\right)=Q_{1}\left(x_{c}\right) w^{\prime \prime}\left(x_{c}, t\right)
\end{gathered}
$$

Substituting the above results into Eq. (40) and integrating over the cross section, we find

$$
m=\frac{I}{I_{r}+\frac{a}{2} I_{c}}
$$

where

$$
I_{r}=\int_{A_{c}} z^{2} d A
$$

and

$$
I_{c}=\int_{A_{c}} \frac{a}{2} z d A
$$

are the second and first moments of the area of the reduced section with respect to $z$ (the origin is at the centroid of the uncracked section).

\section{Application to Beams with a}

\section{Rectangular Cross Section}

The cracked beam theory is used to examine the modes of free vibration of simply supported and cantilevered beams with one single-edge crack. We consider a beam of rectangular cross section of depth $2 d$ and breadth $2 b$, with one crack of depth $a$ located at $x=x_{c}$. The constants $I, I_{c}, I_{r}, K, K_{1}, K_{2}$, $L, L_{1}, L_{2}, L_{3}, L_{\mathbf{4}}, L_{5}$, and $m$ in Eqs. (10), (18), and (44) are

$$
\begin{gathered}
I=\frac{4 b d^{3}}{3}, I_{r}=\frac{2 b\left[(d-a)^{3}+d^{3}\right]}{3}, I_{c}=a b(a-2 d), \\
m=\frac{1}{1+\frac{3}{4}\left(\frac{a}{d}\right)^{2}-\frac{3}{2}\left(\frac{a}{d}\right)-\frac{1}{8}\left(\frac{a}{d}\right)^{3}}, \\
K=0, \quad K^{\prime}=0, \quad K^{\prime \prime}=0, \\
L=C I \exp \left(-2 \alpha \frac{\left|x-x_{c}\right|}{d}\right) \text { where } C=(m-1), \\
L_{1}=I C_{1} \exp \left(-2 \alpha \frac{\left|x-x_{c}\right|}{d}\right),
\end{gathered}
$$




$$
\begin{gathered}
K_{1}=I C_{1} \exp \left(-\alpha \frac{\left|x-x_{c}\right|}{d}\right) \text { where } C_{1}=\left(1-\frac{1}{m}\right), \\
L_{2}=L_{3}=\frac{1}{2} L_{1}^{\prime} ; L_{4}+L_{5}=L_{3}^{\prime}=\frac{1}{2} L_{1}^{\prime \prime} ; K_{2}=K_{1}^{\prime}
\end{gathered}
$$
Eq. (36)

$$
\begin{gathered}
E\left(I+L_{1}-K_{1}\right) Q_{1} w^{\prime \prime \prime}+E\left[2\left(I+L_{1}-K_{1}\right) Q_{1}^{\prime}\right. \\
\left.+\left(2 L_{3}+L_{2}-K_{2}\right) Q_{1}\right] w^{\prime \prime \prime}+E\left[\left(I+L_{1}-K_{1}\right) Q_{1}^{\prime \prime}\right. \\
\left.+\left(2 L_{3}+L_{2}-K_{2}\right) Q_{1}^{\prime}+\left(L_{4}+L_{5}\right) Q_{1}\right] w^{\prime \prime}+\rho A \ddot{w}=0
\end{gathered}
$$

The modes of free vibration of the cracked beam are obtained by assuming simple harmonic motion of frequency, $\omega_{c}$. Taking $w(x, t)=\hat{w}(x) e^{j \omega_{c} t}$ leads to

$$
\begin{gathered}
E\left(I+L_{1}-K_{1}\right) Q_{1} \hat{w}^{\prime \prime \prime \prime}+E\left[2\left(I+L_{1}-K_{1}\right) Q_{1}^{\prime}\right. \\
\left.+\left(2 L_{3}+L_{2}-K_{2}\right) Q_{1}\right] \hat{w}^{\prime \prime \prime}+E\left[\left(I+L_{1}-K_{1}\right) Q_{1}^{\prime \prime}\right. \\
\left.+\left(2 L_{3}+L_{2}-K_{2}\right) Q_{1}^{\prime}+\left(L_{4}+L_{5}\right) Q_{1}\right] \hat{w}^{\prime \prime}-\omega_{c}^{2} \rho A \hat{\hat{w}}=0
\end{gathered}
$$

For an uncracked beam, $Q_{1}$ equal 1 , and $L_{1}, K_{1}, L_{2}, K_{2}$, $L_{3}, L_{4}$, and $L_{5}$ equal 0 . Thus, Eq. (53) reduces to the standard Bernoulli-Euler beam equation.

For a cracked beam, the continuity characteristics of the solution are altered by the crack: the solution has a continuous second derivative $\hat{w}^{\prime \prime}$ but only a piecewise continuous third derivative $\hat{w}^{\prime \prime \prime}$, with a jump at the crack-tip section (for details see [9]). This weaker continuity of the solution significantly deteriorates the convergence of the Rayleigh-Ritz method or the weighted residual method used to estimate the normal modes from Eq. (53).

Free vibration of a simply supported beam

\section{with a mid-span crack}

The modes of free vibration of a simply supported beam with a rectangular cross-section and a mid-span single-edge crack are studied. Since $Q_{1}$ in Eq. (53) is a function of the rate of stress decay, the latter cannot be determined by the above theory alone. Thus, to both validate the theoretical formulation and determine the stress decay rate $\alpha$, numerical results are obtained first from a finite element analysis.

\section{Finite element mesh}

Fig. 2a shows the finite element mesh with four quarterpoint rectangular elements to model the crack tip. Transition elements [17] are used above and below the crack tip elements $[18,19]$ to capture the stress singularity which is assumed to cover the entire thickness of the beam. This mesh is designed to yield accurate results which rapidly converge as the mesh is refined, both for uncracked and cracked beams. It consists of forty 8-noded, plane stress, two-dimensional elements, totaling 151 nodal points and 298 degrees of freedom. In Fig. 2a the beam's slenderness ratio ( $\equiv \frac{l}{2 d}$ ) is equal to 20.0 . The 16 nodal displacements for each element are the in-plane displacements $u$ and $w$ at each node. The size of the quarter-point elements at the crack tip is chosen to capture the effect of the singularity. The elements cover $\frac{1}{24}$ of the beam's length in the axial direction, such that they extend over nearly all the stress concentration. Quarter-point elements of various sizes were tested, such that the elements' length was much smaller or much greater than the range of the stress concentration. Too narrow or too wide crack-tip elements led to considerable errors. It was demonstrated numerically that the finite element mesh shown in Fig. 2a gives a nearly optimal result for the present problem. Since no special procedure is needed to compute the stiffness and mass matrices for the distorted crack tip element, any general purpose finite element code can be used.

Fig. 2b illustrates an al ternative finite element mesh, which essentially replaces every rectangular quarter-point element in Fig. 2a by two triangular quarter-point elements. The mesh consists of 44 elements, 157 nodal points and 310 degrees of dreedom. All the results obtained by the triangular elements are very close to those given by the rectangular ones.

To validate the finite element model, the lowest three natural frequencies of the uncracked beam were compared to Bernoulli-Euler theory results. As shown in Table 1, the finite element frequencies are respectively $0.24,0.96$, and $1.8 \%$ lower than the Bernoulli-Euler results. Since there are no geometrical assumptions for the finite element formulation, the natural frequencies are expected to be lower, especially for the higher modes.

\section{Finite element results}

The modes of vibration were computed for crack depths of $\frac{1}{4}, \frac{1}{3}$, and $\frac{1}{2}$ of the total beam thickness. The natural frequencies, as shown in Table 1 and Figs. 3 and 4, are presented in the form of the frequency ratio (FR), the ratio of the frequency of the cracked beam to that of the uncracked beam, against the crack depth ratio (CR), the ratio of the depth of the crack to the beam thickness. The first three mode shapes for cracked beams with crack ratios of $\frac{1}{4}, \frac{1}{3}$, and $\frac{1}{2}$ are plotted in Figs. 57 and compared to the modes of the uncracked beam. The changes in the first and third mode shapes are significant for large crack ratios only ( $\left.C R>\frac{1}{4}\right)$. The second mode shapes are unaffected by the crack for all ratios examined. This is because the crack is located at mid-span, where compressive or tensile stresses equal zero in the even vibration modes. Therefore, for a beam pinned at both ends, a single-edge crack at the middle will not affect the even, antisymmetrical modes of vibration.

It has been shown in Ref. [9] that for a pair of cracks, the strain energy in the odd modes decreases, while in the even modes it remains unchanged. Similarly, for single-edge cracked beams, Table 1 shows that the strain energy in the first and third modes decreases as the crack depth increases and that the strain energy for the second mode remains unchanged. This is consistent with the above frequency and mode shape observations.

\section{Galerkin procedure}

Because the modes of the cracked beam have a discontinuous third derivative, their Galerkin expansion in a series in

the infinitely differentiable modes of the uncracked beam [9],

$$
\hat{w}(\xi)=\sum_{i=1}^{N} a_{i} \sin (i \pi \xi)
$$

requires at least 100 terms $(N \geq 100)$ to satisfy the convergence criterion

$$
U^{N}=\max _{i=1,2,3}\left|\frac{\Delta \omega_{i}^{N}}{\omega_{c i}^{N}}\right|<\epsilon
$$

where $\Delta \omega_{i}^{N}$ is the change in the $i$-th frequency from the $N$ term to the $(N+1)$-term calculation, $\omega_{c i}^{N}$ is the $N$-term estimate of the $i$ th frequency of the cracked beam, and $\epsilon$ is a small real number. For all cases presented in this paper, convergence is considered to be achieved when the relative frequency change is less than $\epsilon=2.0 \times 10^{-5}$.

Substituting Eq. (54) into Eq. (53) and applying the Galerkin procedure, we obtain a discrete eigenvalue problem of size $N$ in the generalized coordinates, $a_{i}$ :

$$
\left[K_{\mathrm{c}}\right] \underline{a}-\omega_{\mathrm{c}}^{2}\left[M_{\mathrm{c}}\right] \underline{a}=\underline{0}
$$


where

$$
\left[M_{c}\right]=\frac{\rho A l}{2}[I]
$$

is the mass matrix, $[I]$ is the identity matrix, the vector $\underline{a}$ is defined as $\left[a_{1}, a_{2}, \ldots ., a_{N}\right]^{T}$, and

$$
\begin{aligned}
{\left[K_{c}\right]=} & \frac{E I \pi^{4}}{l^{3}} \int_{0}^{1}\left\{i^{2} j^{2}\left(1+\bar{L}_{1}(\xi)-\bar{K}_{1}(\xi)\right) Q_{1}(\xi) \sin i \pi \xi \sin j \pi \xi\right. \\
& +\frac{1}{2} i j^{2}\left[\left(\bar{L}_{2}(\xi)-\bar{K}_{2}(\xi)\right) Q_{1}(\xi)\right] \cos i \pi \xi \sin j \pi \xi \\
+ & \left.\frac{1}{2} i^{2} j\left[\left(\bar{L}_{2}(\xi)-\bar{K}_{2}(\xi)\right) Q_{1}(\xi)\right] \sin i \pi \xi \cos j \pi \xi\right\} d \xi
\end{aligned}
$$

is the stiffness matrix. The functions $\bar{L}(\xi), \bar{L}_{1}(\xi), \bar{K}_{1}(\xi), \bar{L}_{2}(\xi)$, $\bar{K}_{2}(\xi)$, and $Q_{1}(\xi)$ are defined as

$$
\left\{\begin{array}{l}
\bar{L}(\xi)=C \exp \left(-2 \alpha \frac{\left|\xi-\frac{1}{2}\right| l}{d}\right) \\
\bar{L}_{1}(\xi)=C_{1} \exp \left(-2 \alpha \frac{\left|\xi-\frac{1}{2}\right| l}{d}\right), \\
\bar{K}_{1}(\xi)=C_{1} \exp \left(-\alpha \frac{\left|\xi-\frac{1}{2}\right|}{d}\right), \\
\bar{L}_{2}(\xi)=\frac{d L_{1}(\xi)}{d \xi}, \quad \bar{K}_{2}(\xi)=\frac{d K_{1}(\xi)}{d \xi}, \\
Q_{1}(\xi)=\frac{1+L_{1}(\xi)-K_{1}(\xi)}{1+L(\xi)}
\end{array}\right.
$$

\section{Determination of the stress decay constant $\alpha$}

Once the number of terms yielding satisfactory convergence is determined, the rate of stress decay $\alpha$ is obtained by fitting the natural frequencies calculated by Galerkin's method best with the finite element results, in a least square sense. Only the fundamental frequency is considered for simplicity.

The fundamental frequency drop in terms of crack depth is shown in Fig. 3 and Table 1. The least square fit of the 100-term Galerkin solution with the finite element results determined the rate of stress decay $\alpha$ to be 1.979 . As we expect, this value should be very close to 1.936 that was determined for beams with symmetric cracks (the discrepancy can be considered as a computational error). This is based on the physical argument that the normal stress decays at the same rate for both symmetric and single cracks, because the stress decay rate is one of the beam's material properties. A similar argument was made for the static problem in fracture mechanics $[15,16]$. Examination of the mode shapes

The mode shapes obtained by the Galerkin and finite element formulations are compared in Fig. 5-7. One observes that Galerkin results are consistently in good agreement with the finite element ones.

\section{Combination of the higher modes information}

The prediction of a crack's location and depth based upon only one mode could be misleading. For instance, by reviewing only the data for second mode, as given in Table 1 and Fig. 6 , one would conclude that the beam is not damaged. This implies that different modes viewed separately might yield different predictions of damage, i.e., crack position and depth. Moreover, from Figs. 5(c) and 7(c), the effect on the third mode is more severe than that on the first. Therefore, it is expected that a multi-mode analysis would be needed to determine the position and size of the crack.

Fig. 4 and Table 1 show the variation of the third natural frequency with the crack ratio. Similar to the fundamental mode, there is excellent agreement between Galerkin and finite element results for crack ratios smaller than $\frac{1}{2}$. Crack ratios larger than $\frac{1}{2}$ were not considered, as failure would occur before such a value is reached.

\section{Free vibration of cracked cantilevered beams}

The cracked beam theory derived in the previous section is applied to a cantilevered beam (see Fig. 8). However, the numerical integration of the free bending modes of the uncracked beam, required to generate the mass and stiffness coefficients in the Galerkin procedures, causes a computer overflow, because these modes involve hyperbolic functions and many modes are required. Therefore, the Galerkin procedure with 100 terms is impractical in the cantilevered case.

Local Ritz method

To circumvent this problem, a local Rayleigh-Ritz approach which uses a piecewise fit to the deflection shape is presented. The displacement, $\hat{w}(x)$, is approximated by piecing cubic polynomials, each defined over only a portion of the structure, or subbeam. The coefficients of the cubic polynomials can be determined uniquely in terms of the displacements and slopes at the end points. The displacement at a point within the $i$ th subbeam is approximated as

$$
\hat{w}_{i}(\eta)=\underline{F}^{T}(\eta) \underline{u}_{i},
$$

where $\underline{E}=\left[F_{1}, F_{2}, F_{3}, F_{4}\right]^{T}$ is a vector of prescribed (shape) functions of position and $\underline{u}_{i}$ is a vector of end displacements and slopes for the $i$-th subbeam. The shape functions $\left(F_{j}\right)_{j=1, \ldots, 4}$ are listed in the Appendix. This piecewise polynomial interpolation amounts to a finite element solution of the cracked beam differential equation (53). In this example, a local RayleighRitz model with four shape functions, $M$ identical subbeams, $M+1$ nodes, and $2 M$ DOF is used for the analysis.

The free vibration eigenvalue problem is expressed as

$$
\left[K_{e}\right] \underline{u}-\omega_{c}^{2}\left[M_{e}\right] \underline{u}=\underline{0}
$$

where $\underline{u}$ is the vector of nodal displacements, and $\left[K_{e}\right]$ and $\left[M_{e}\right]$ are $(2 M \times 2 M)$ stiffness and mass matrices for the entire beam. The assemblage process to obtain $\left[K_{e}\right]$ and $\left[M_{e}\right]$ is symbolically described by

$$
\left(\underline{u},\left[K_{e}\right],\left[M_{e}\right]\right)=\sum_{i=1}^{M}\left(\underline{u}_{i},\left[k_{i}\right],\left[m_{i}\right]\right)
$$

where $\underline{u_{i}},\left[k_{i}\right]$ and $\left[m_{i}\right]$ are the nodal displacements, stiffness and mass matrices, respectively, for the $i$-th subbeam, and the summation is over all $M$ subbeams. The $(4 \times 4)$ mass and stiffness matrices of the $i$ th subbeam in the local coordinate system are

$$
\left[m_{i}\right]=\int_{0}^{l_{s}} E^{T} E d \eta
$$

and

$$
\begin{aligned}
{\left[k_{i}\right]=} & \frac{E I}{\rho A} \int_{0}^{l_{0}}\left\{\left(1.0+\bar{L}_{1}(\eta)-\bar{K}_{1}(\eta)\right) Q_{1}(\eta) \underline{B}^{T} \underline{B}\right. \\
& +\frac{1}{l}\left[\left(\bar{L}_{2}(\eta)-\bar{K}_{2}(\eta)\right) Q_{1}(\eta)\right] \underline{B 1}^{T} \underline{B} \\
& \left.+\frac{1}{l}\left[\left(\bar{L}_{2}(\eta)-\bar{K}_{2}(\eta)\right) Q_{1}(\eta)\right] \underline{B}^{T} \underline{B 1}\right\} d \eta
\end{aligned}
$$

where $\underline{B 1}=\frac{d}{d \eta} \underline{F}$ and $\underline{B}=\frac{d^{2}}{d^{2} \eta} \underline{F}$, the so-called strain-displacement vector.

The value of $\alpha$ was determined to be 1.930 by a least square fit with the finite element results (see Fig. 9). The eigenvalue problem (61) was then solved for increasing number of subbeams, $M$, until a frequency convergence test was satisfied. The frequency convergence criterion used in the present example was similar to that used in the Galerkin approach, Eq. (55), except that here the number of subbeams, $M$, is increased instead of the number of uncracked modes, $N$. At least 50 subbeams $(M \geq 50)$ were needed in the local Rayleigh-Ritz procedure to satisfy the convergence criterion $\left(\epsilon=2.0 \times 10^{-5}\right)$ 
for the fundamental mode.

\section{Experimental verification}

The effects of cracks on the natural frequencies of beams have been studied experimentally by Wendtland [20] and Wendtland and Wiederuh [21]. In Ref. [20], the cracks were modeled by sawing cuts of width 0.0035 times the length of the beam (see Fig. 8). The five lowest natural frequencies were obtained experimentally for various crack ratios and positions. Here, we compare our theoretical results with the experimental ones in Ref. [20].

We obtained the lowest five eigenfrequencies for rectangular beams with cracks at $\frac{x_{c}}{\tau}=X C=0.2,0.3,0.55,0.6,0.7$, and 0.8 . Selected theoretical results are compared with the experimental data obtained by Wendtland [20] in Figs. 9 to 14. The data is presented in the form of the frequency ratio (FR) versus the crack depth ratio (CR). Observe that, our theoretical frequencies correlate very closely with the experimental ones for crack ratios up to 0.8 . This excellent comparsion confirms the validity of our theory.

Examination of the mode shapes

The first five mode shapes of cracked beams with crack ratios of $0.13,0.5$, and 0.8 and various locations are compared to those of an uncracked beam in Figs. 15 to 19. Observe the severe deformation near the crack tip for large cracks, which can be used to detect crack position.

Effects of crack position on the dynamical response of a cracked beam

The effect of crack position on the natural frequencies and mode shapes has been discussed in Ref. [9] and section 3.1 for a simply supported beam with cracks at mid-span. In both cases it was shown that only the odd, symmetrical, modes are affected by cracks. As was indicated earlier, it is clear that to have an adequate basis to estimate damage from frequency and mode shape information, a multi-mode analysis should be considered.

Similar conclusions can be drawn from the analysis of a cantilevered beam with a single-edge crack. The first bending frequency is shown in Fig. 20 as a function of the crack ratio for four crack positions, $X C=0.2,0.4,0.6$, and 0.8 . The drop in frequency is greater for cracks near the clamped end, while the frequency is almost unchanged when the crack is located near the free end. This result can be explained by noting that the compression and tension, or the bending moment, are distributed heavily near the fixed end for the fundamental mode, leading to a severe loss in bending stiffness due to the crack. However, this drop in frequency does not occur in the higher modes. The solid curves in Figs. 21 and 22 show that the second and third frequencies are comparatively much less affected for $C R=0.2$, but are strongly affected for different crack locations $(X C=0.6$ and 0.8 for the second and third modes, respectively). In order words, the frequency drop is greatest for a crack located where the bending moment is largest. Clearly, the sensitivity to cracks depends highly on the mode number and crack position.

Figs. 23 and 24 are for the same crack depths as Figs. 17 and 18 , but for slightly different crack positions $(X C=0.4$ instead of 0.3 and 0.5 ). One observes that the effect of the crack on the mode shapes is somewhat different from that in Figs. 17 and 18 , such that the modes are less altered by the crack. Thus, the sensitivity of the modes of vibration to cracks can very substantially depending on the crack position. This provides an indication of how the effect on the mode shapes can provide useful information in the crack identification procedure.

Several observations can be made from the above discussion. First, for a specific mode, the effects on the bending frequency and mode shapes of a cracked beam become more severe as crack depth grows. Second, for a certain crack ratio, crack position strongly affects the dynamic behavior of a cracked beam. Third, if the position of the crack is known information, one specific mode may be sufficient to obtain accurate results in the crack identification problem. Finally, if the crack position is unknown, the uniqueness and accuracy of the identification process becomes questionable. But in general, the more modes are used for crack identification, the more accurate and reliable the result will be.

\section{Conclusions}

A theory for the flexural motion of a Bernoulli-Euler beam containing a single-edge crack is presented. It is based on two key kinematic assumptions made to satisfy the compatibility requirements in the vicinity of the crack. First, the stress concentration near the crack tip is accounted for by introducing a crack function in to the beam's compatibility relations. Second, a function is introduced that modifies the in-plane displacement and its slope to avoid a discontinuity in the slope of the neutral axis and in the axial displacement at the crack, which occurs with a non-symmetric crack configuration.

The equation of motion and associated boundary conditions are derived. The validity of the theory is established by studying two different sets of boundary conditions. The analytical solutions show excellent agreement with both experimental results and finite element predictions. The effects of cracks on frequency and mode shape are found to be very sensitive to crack location and mode number.

The present theory could be extended easily to beams with nonrectangular cross section and account for shear deformation (Timoshenko beam). Other future work includes the development of an inverse analysis procedure to identify the cracks properties from dynamical measurements.

\section{Acknowledgements}

The authors are grateful to Professor John Taylor (The University of Michigan) for his valuable comments and expert suggestions. This work was partly supported by NSF Grant No. MSM- 8700820 .

\section{References}

[1] T. G. Chondros and A. D. Dimarogonas $1980 \mathrm{~J}$. of Sound and Vibration 69 531-538. Identification of Cracks in Welded Joints of Complex Structures.

[2] A. D. Dimarogonas and G. Massouros 1980 Engng. Fracture Mech. 15 439-444. Torsional Vibration of a Shaft with a. Circumferential Crack.

[3] A. D. Dimarogonas and C. A. Papadopoulos $1983 \mathrm{~J}$. of Sound and Vibration 91 583-593. Vibration of Cracked Shafts in Bending.

[4] P. Gudmundson 1983 J. Mech. Phys. Solids 31 No.4 329345. The Dynamic Behaviour of Slender Structures with Cross-Sectional Cracks.

[5] C. A. Papadopoulos and A. D. Dimarogonas 1987 Ingenieur-Archiv 57 257-266. Coupling of Bending and Torsional Vibration of a Cracked Timoshenko Shaft. 
[6] P. Cawley and R. D. Adams 1979 J. Strain Anal. 14 49-57. The Location of Defects in Structures from Measurements of Natural Frequencies.

[7] P. Cawley and R. D. Adams 1979 ASME paper 79-DET46. Defect Location in Structures by A Vibration Technique.

[8] S. Christides and A. D. S. Barr 1984 Int. J. Mech. Sci. 26 No. 11/12 639-648. One-Dimensional Theory of Cracked Bernoulli-Euler Beams.

[9] M. H. Shen and C. Pierre $1990 \mathrm{~J}$. of Sound and Vibration 137 No. 2. Natural Modes of Bernoulli-Euler Beams with Symmetric Cracks, to appear.

[10] L. B. Freund $1972 J$. of Applied Mechanics 39 Trans. ASME 94 Series E 601-602. The Initial Wave Front Emitted by a Suddenly Extending Crack in an Elastic Solid.

[11] L. B. Freund $1972 J$. of the Mechanics and Physics of Solids 20 129-152. Crack Propagation in an Elastic Solid Subjected to General Loading.

[12] L. B. Freund $1974 \mathrm{~J}$. of the Mechanics and Physics of Solids 22 137-146. Crack Propagation in an Elastic Solid Subjected to General Loading.

[13] S. Bodner $1973 \mathrm{~J}$. of the Mechanics and Physics of Solids 21 1-8. Stress Waves Due to Fracture of Glass in Bending.

[14] L. B. Freund and G. Herrmann 1975 J. of Applied Mechanics 43 112-116. Dynamic Fracture of a Beam or Plate in Pure Bending.

[15] G. R. Irwin 1960 Structural Mechanics, Edited by P. C. Goodier and N. J. Hoff, Pergamon Press, 557. Fracture Mechanics section.

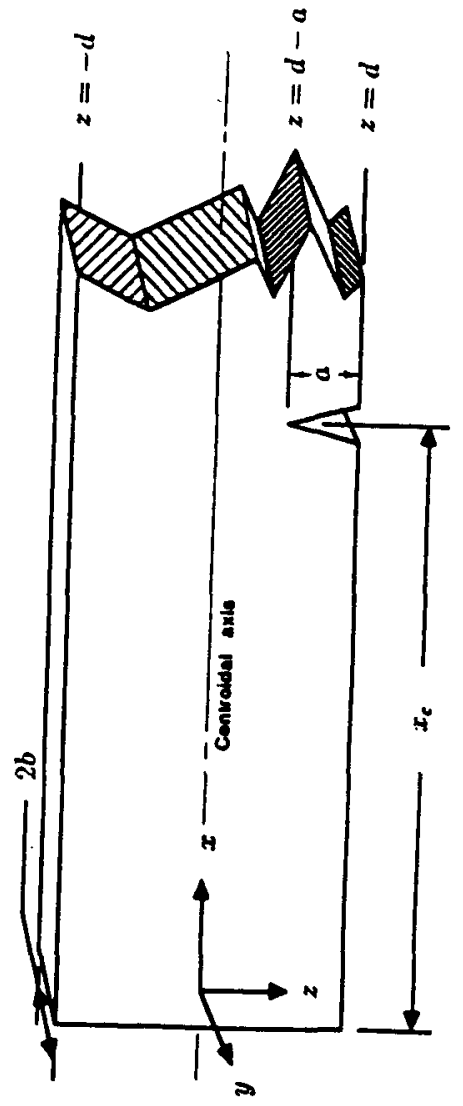

[16] P. C. Paris and G. C. Sih 1965 Fracture Toughness and its Applications ASME STP- 381 30. Stress Analysis of Cracks.

[17] P. P. Lynn and A. R. Ingraffea 1977 Int. J. Num. Meth. in Engrg. 11 1031-1036. Transition Elements to be Used with Quarter-Point Crack-Tip Elements.

[18] R. D. Henshell and K. G. Shaw 1975 Int. J. Num. Meth. Engng. 9 495-509. Crack Tip Finite Element are Unnecessary.

[19] R. S. Barsoum 1976 Int. J. Num. Meth. Engng. 10 2537. On the Use of Isoparametric Finite Element in Linear Fracture Mechanics.

[20] D. Wendtland 1972 Anderung der Biegeeigen Frequenzen Einer Idealisierten Schaufel Durch Risse, Ph.D. Thesis, University of Karlsruhe.

[21] D. Wendtland and E. Wiederuh 1974 Forsch. Ingenieurwes 40 60-66. Anderungen der Torsionseigenfrequenzen von Turbomaschineschaufeln Durch Risse.

\section{Appendix}

$$
\begin{gathered}
F_{1}(\eta)=1-3\left(\frac{\eta}{l_{s}}\right)^{2}+2\left(\frac{\eta}{l_{s}}\right)^{3} \quad F_{2}(\eta)=\eta-2 \frac{\eta^{2}}{l_{s}}+\frac{\eta^{3}}{l_{s}^{2}} \\
F_{3}(\eta)=3\left(\frac{\eta}{l_{s}}\right)^{2}-2\left(\frac{\eta}{l_{s}}\right)^{3} \quad F_{4}(\eta)=-\frac{\eta^{2}}{l_{s}}+\frac{\eta^{3}}{l_{s}^{2}} \\
l_{s}=\frac{l}{M}, \quad 0 \leq \eta \leq l_{s}
\end{gathered}
$$
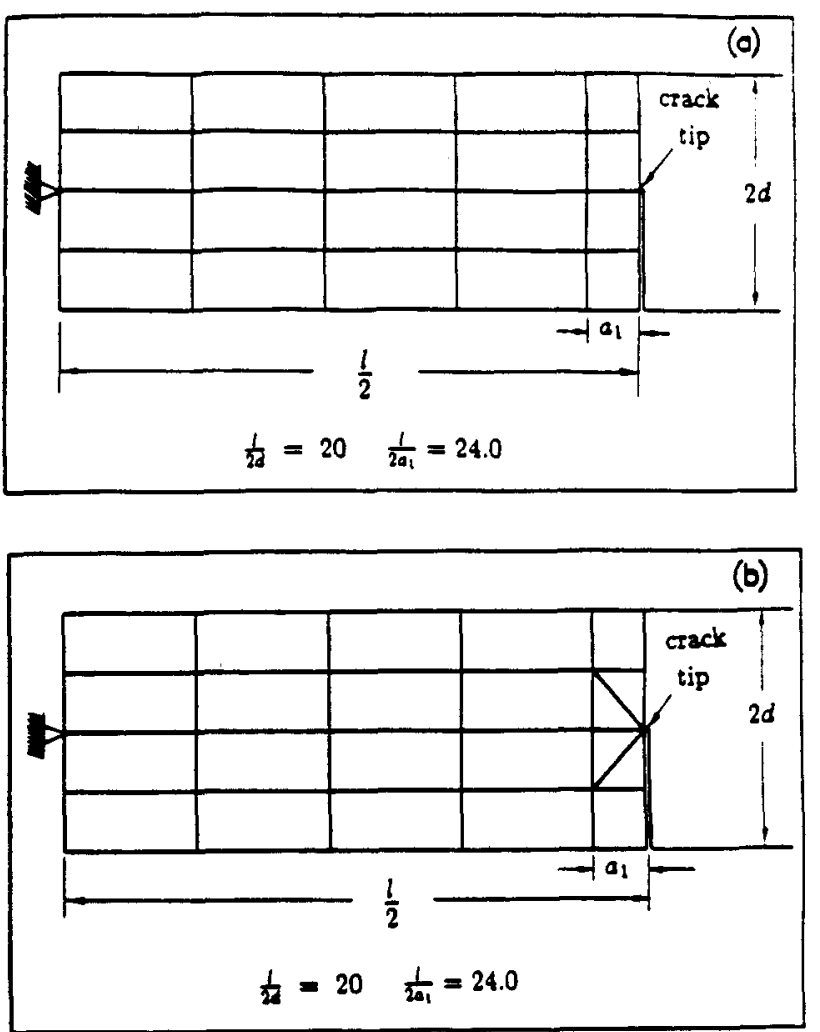

Figure 2. Finite element gridwork for a simply supported beam conaining a single edge cracks at mid-span. $x_{c}=\frac{1}{2}$ 


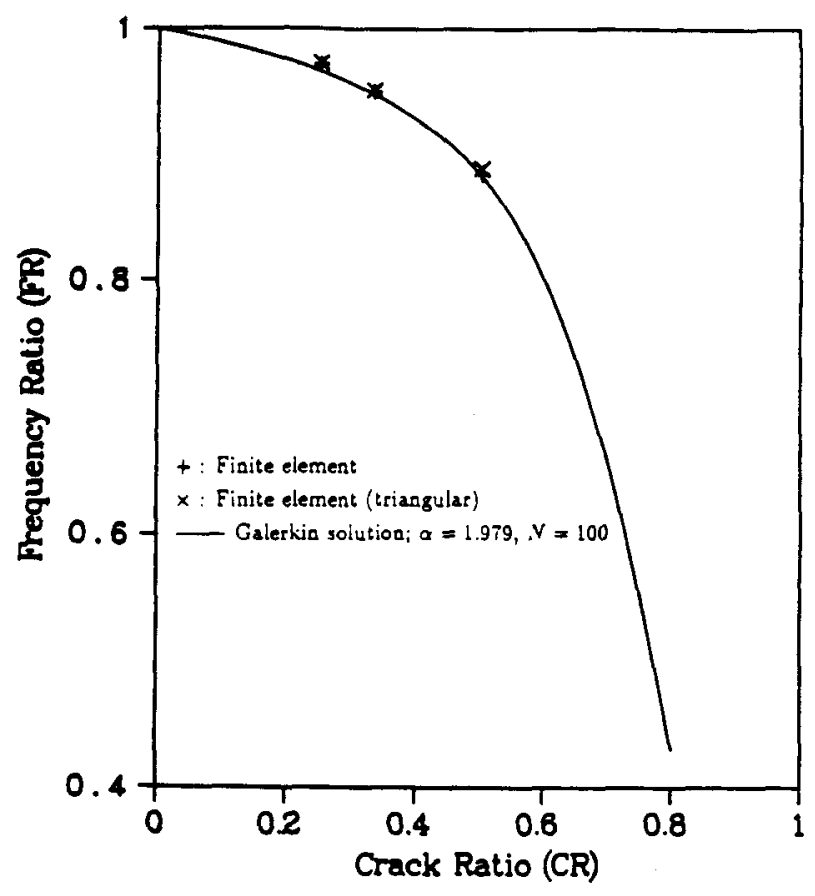

Figure 3. Fundamental natural frequency in terms of crack depth. Theoretical and finite element results are shown for a simpiy supported beam $\left(\frac{1}{28}=20\right)$ with a single-edge crack at mid-span $\left(x_{c}=\frac{1}{2}\right)$.

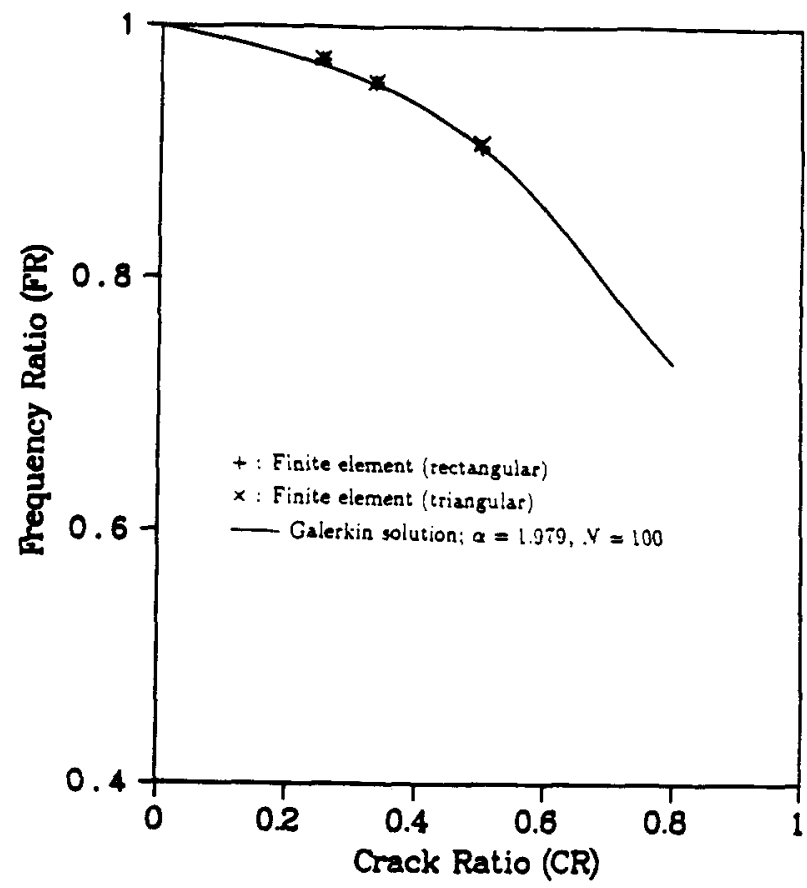

Figure 4. Third natural frequency in terrns of crack depth. Theoretical and finite element results are shown for a simply supported beam $\left(\frac{1}{2 c}=20\right)$ with a singleedge crack at mid-span $\left(\boldsymbol{x}_{c}=\frac{1}{3}\right)$.
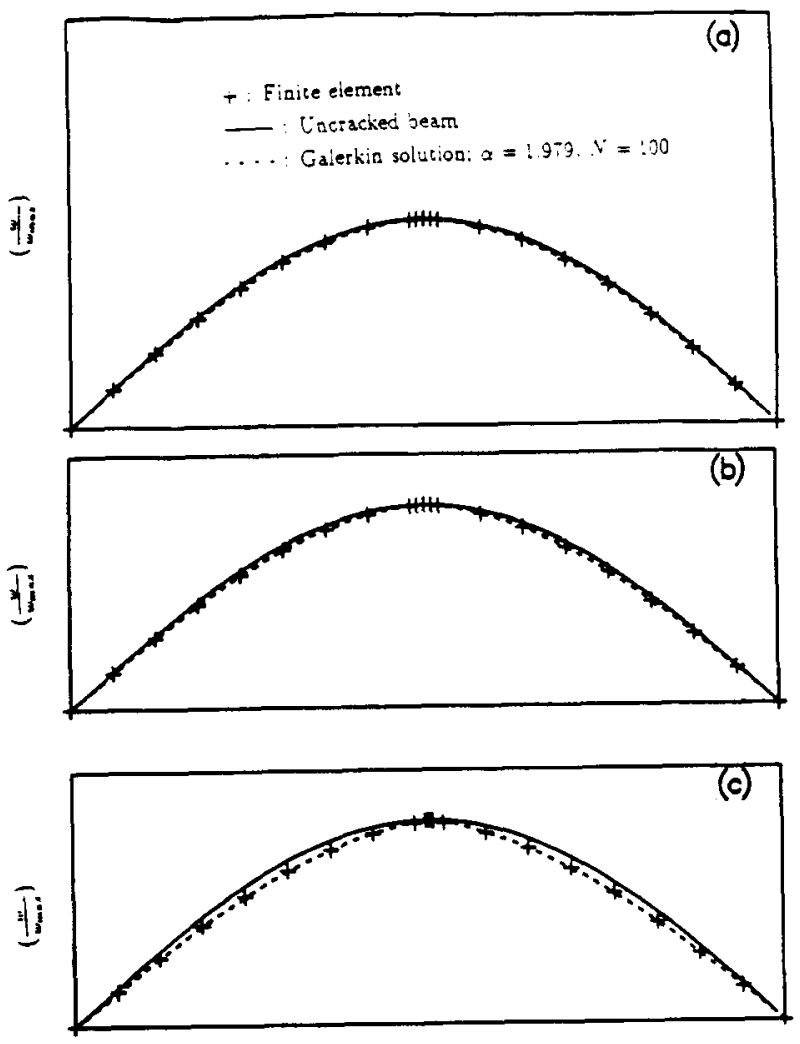

Figure 5. First mode shape of a simply supported beam $\left(\frac{i}{3 \alpha}=20\right.$ ) with a single-edge crack at mid ipan $\left(x_{c}=-i\right)$ Galerkin and finite element results are shown for various cracix tatios: (a) $\mathrm{CR}=\frac{1}{4}$, ib) $\mathrm{CR}=\frac{1}{3}$. (c) $\mathrm{CR}=\frac{1}{2}$.
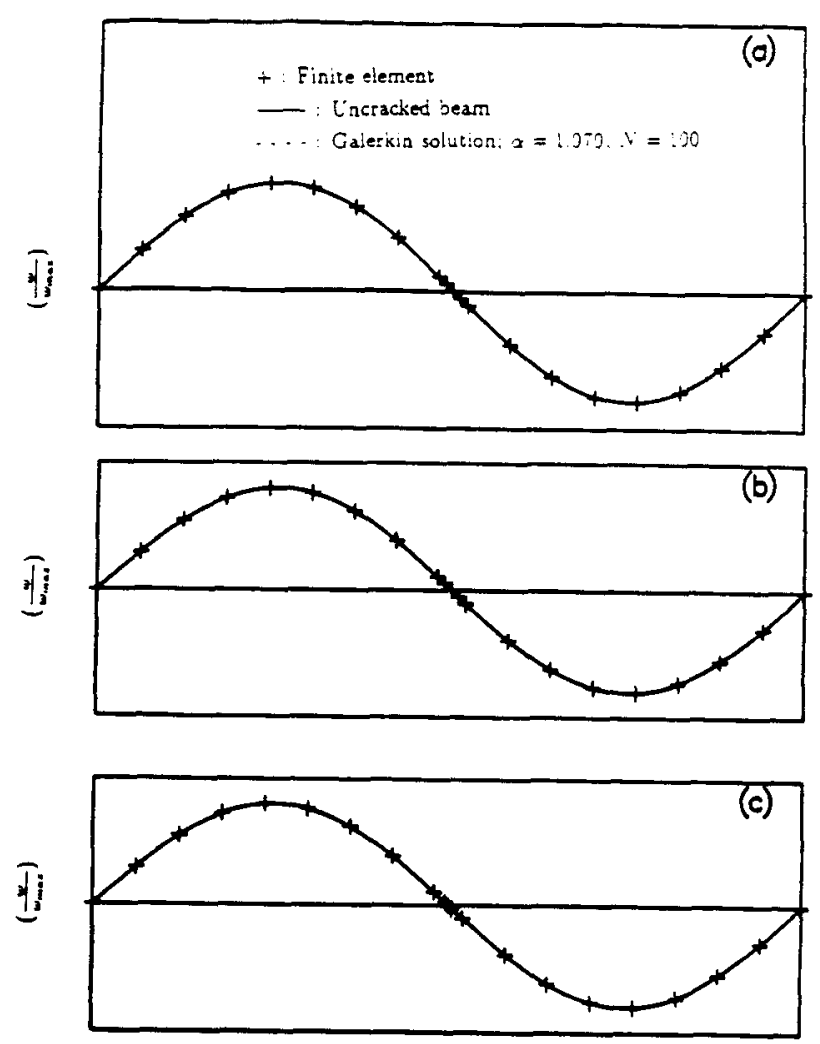

Figure 8. Second mode shape of a simply supported beam $\left(\frac{1}{2 d}=20\right)$ with a single-edge crack at mid-span $\left(x_{c}=\frac{1}{3}\right)$. Galerkin and finite element results ate shown for various crack ration: (a) $C R=\frac{1}{4}$, (b) $C R=\frac{1}{3}$, (c) $C R=\frac{1}{3}$ 

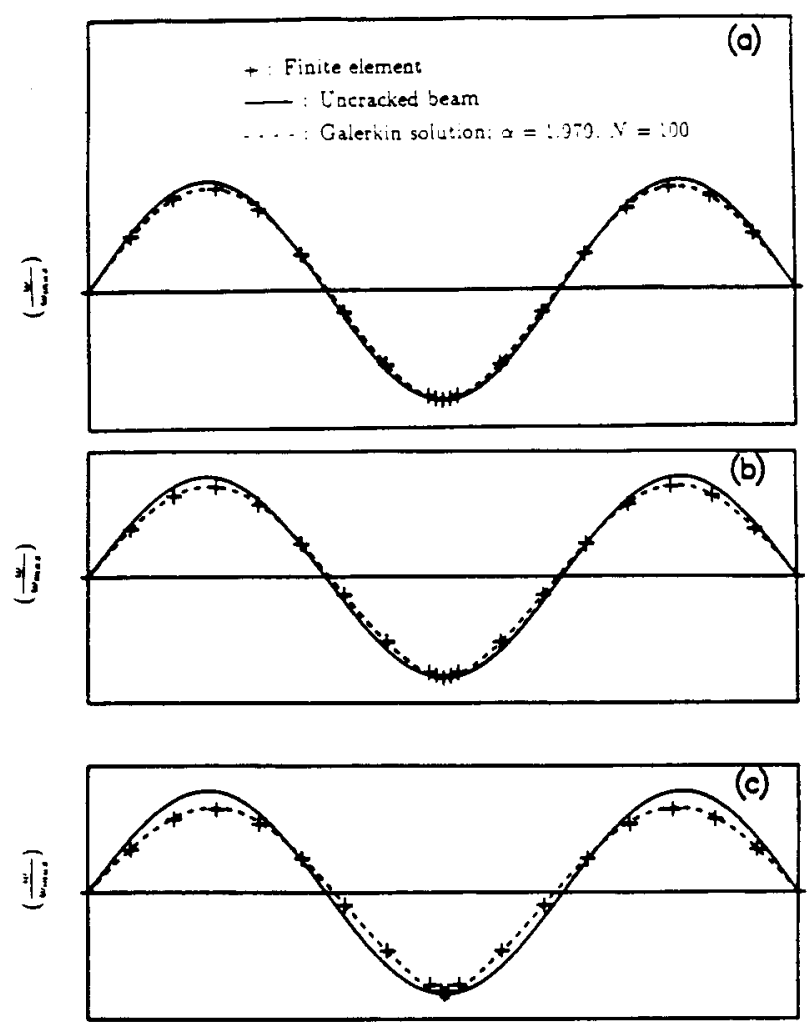

Figure 7 . Third mode shape of a simply supported beam $\left(\frac{1}{3 d}=20\right)$ with a single-edge crack at mid-apan $I_{0}=-1$. Galerkin and finte element results are shown for various crack satios: $(a) C R=\frac{1}{4}$, (b) $C R=\frac{1}{3}$, ic $) C R=\frac{1}{2}$.

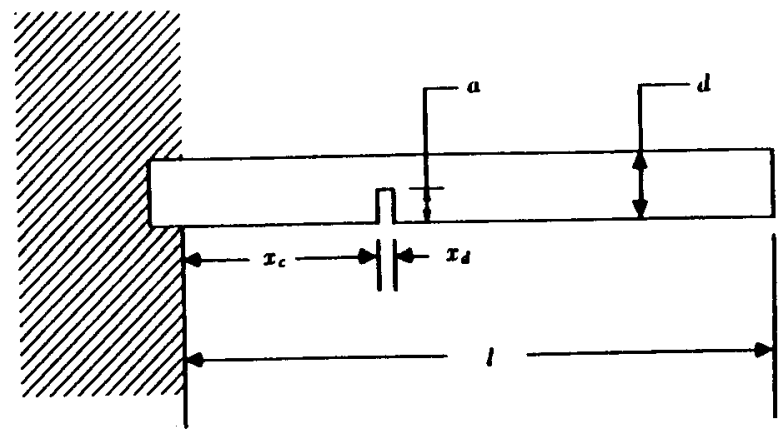

Figure 8. (Eeonetry of the experimental cantitevered bean with a single edge crack

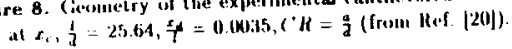

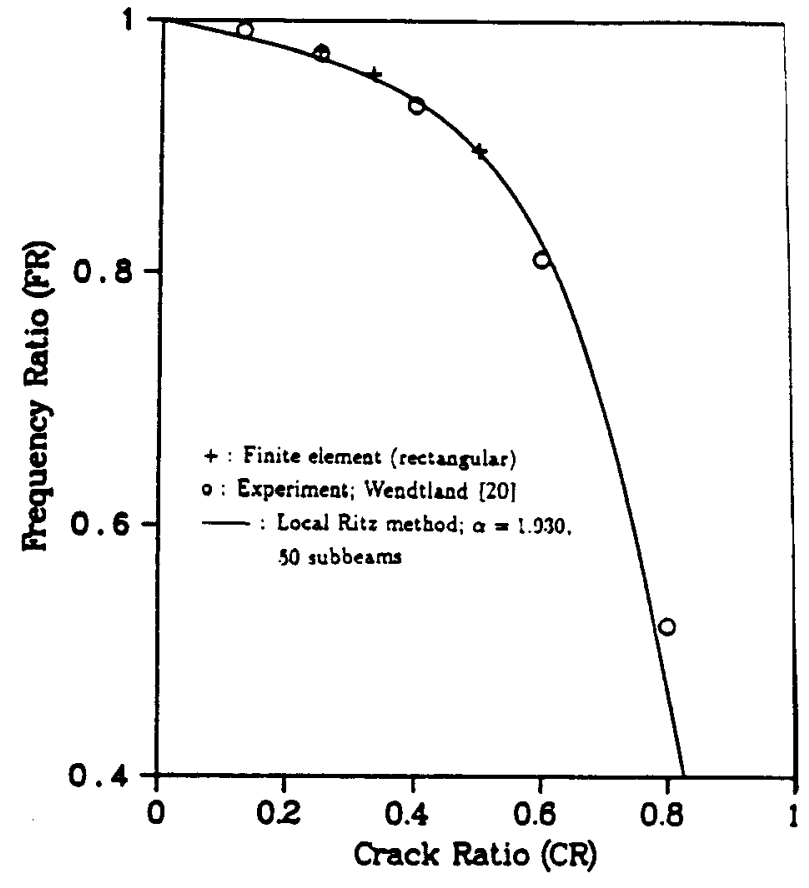

Figure 9. Fundamental natural frequency in terms of crack depth. Theoretical, finite element, and experimental tesults are show for a cantilevered beam $\left(\frac{1}{2 g}=25.64\right)$ with a single-edge crack at $x_{c}=0.2 l(X C=0.2)$.

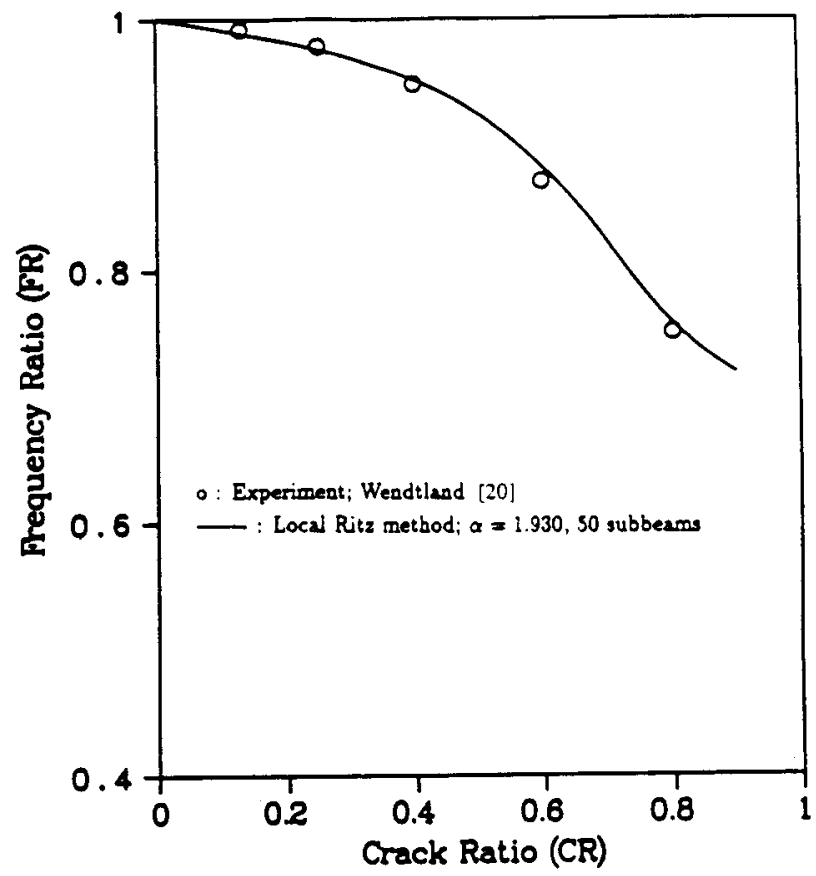

Figure 10. Third atural frequency in terms of crack depth. Theoretical and experimental results are shown for a cantilevered beam $\left(\frac{i}{2 d}=25.64\right)$ with a single-edge crack at $x_{r}=0.3 u(X C=0.3)$ 


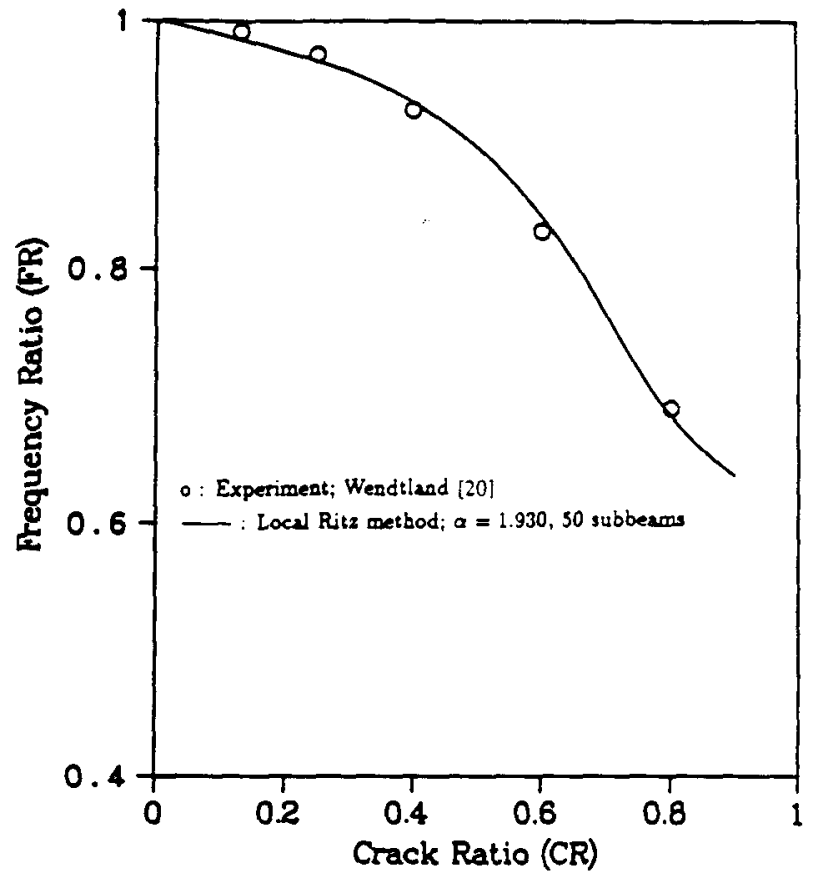

Figure 11. Third natural frequency in terms of crack depth. Theoretical and exper imental results are shown for a cantilevered beam $\left(\frac{i}{3 d}=25.64\right)$ with a single-edge crack at $x_{c}=0 . \vec{i}(X C=0 . i)$

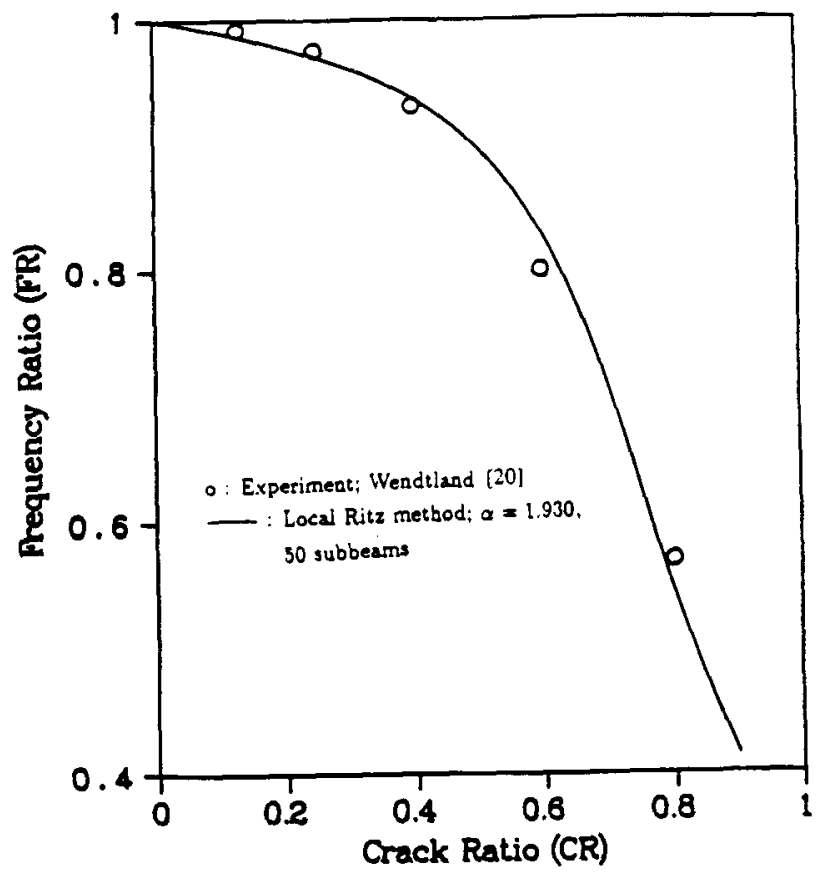

Figure 12. Second natural frequency in terms of crack depth. Theoretical and expesgure 12. Second natural trequency in terms of crack depth. Theortica angle-edge imental results are shown for a canct

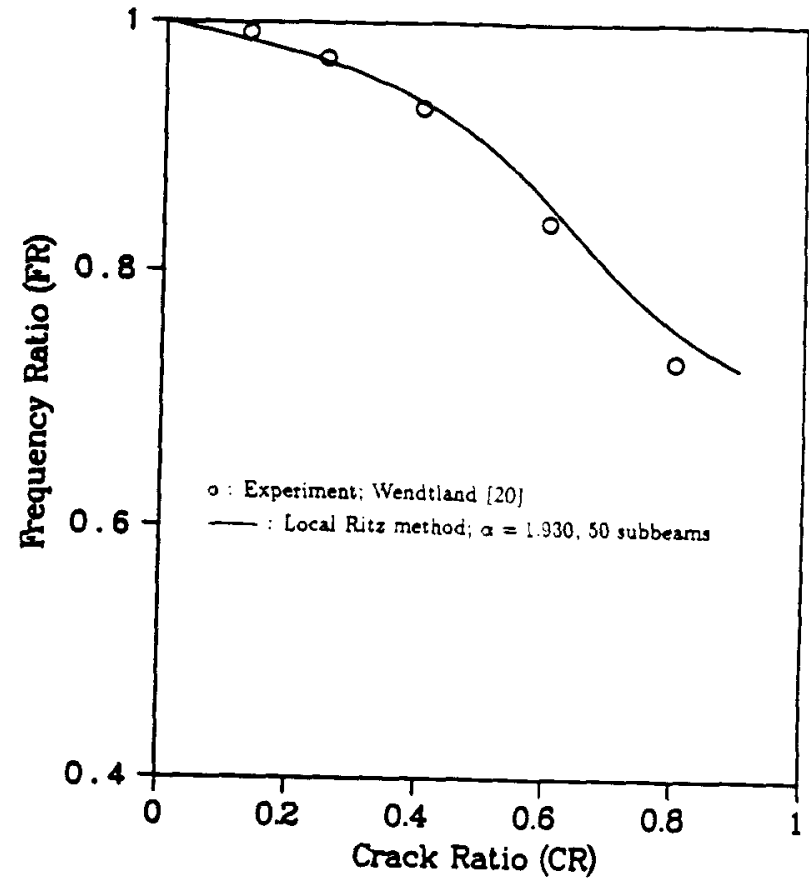

Figure 13. Fourth natural frequency in terms of crack depth Theoretical and exper irnental results are show for a cantilevered beam $\left.\frac{1}{3 a}=25.64\right)$ with a single-edge crack at position $x_{c}=0.84(X C=0.8)$

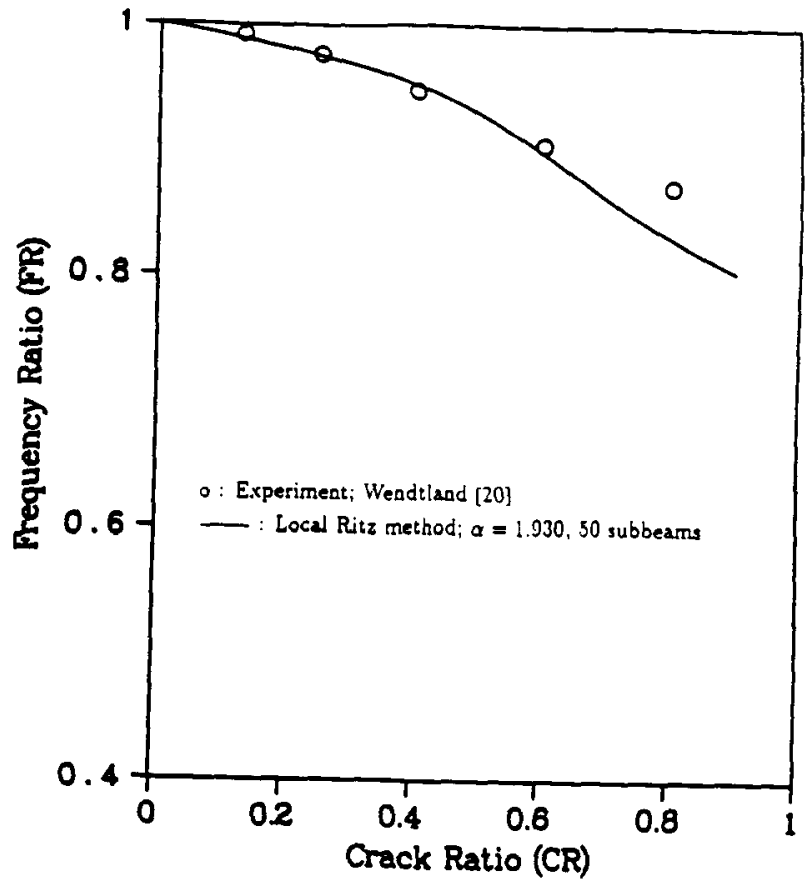

Figure 14. Fifth natural frequency in terms of crack depth. Theoretical and experi. mental results are shown for a cantilevered beam $\left(\frac{1}{2 g}=25.64\right)$ with a single-edge crack at position $x_{\epsilon}=0.6 l(X C=0.6)$. 


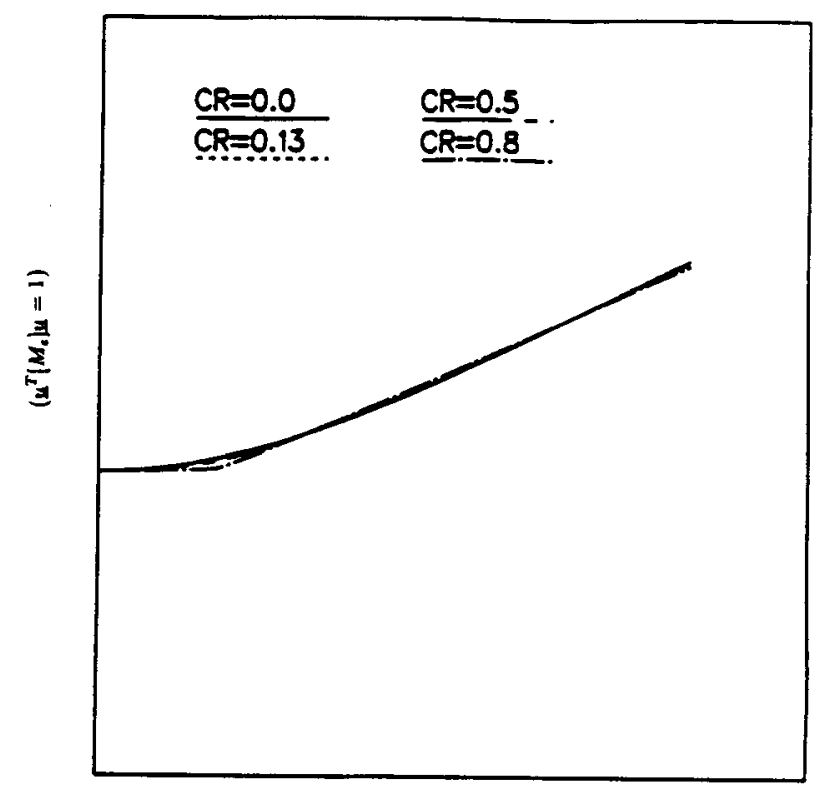

Figure 13. First mode shape of a cantilevered beam $\left(\frac{t}{2 d}=25.64\right)$ with a single-edge crack at $x_{c}=0.2 l(X C=0.2)$. Ritz's result is shown for various track ratios: $C R=0.0,0.13,0.5,0.8$

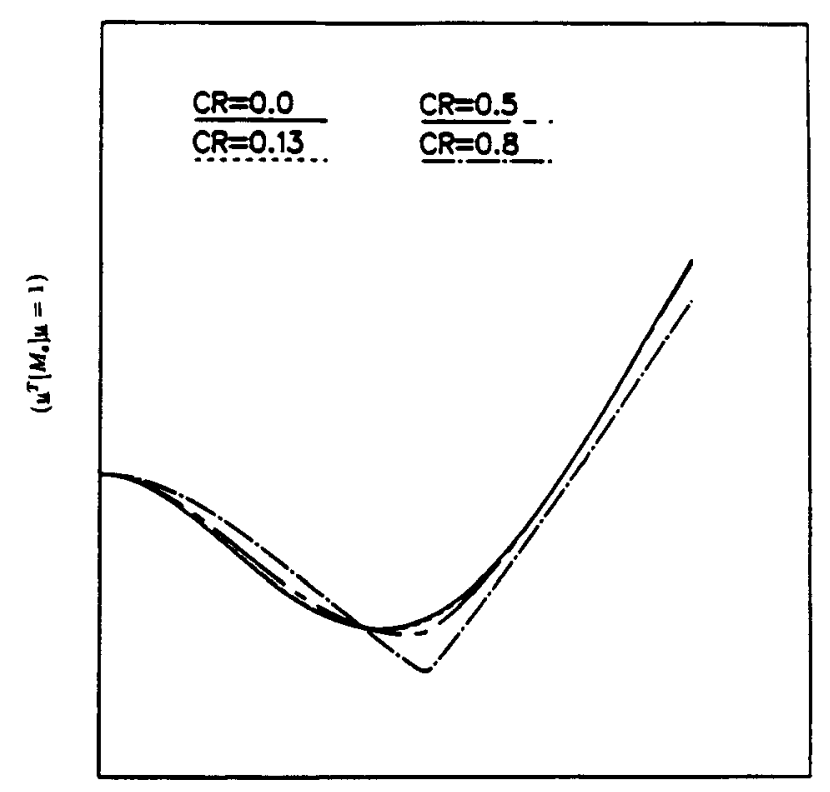

Figure 16. Second mode shape of a cantilevered beam $\left(\frac{1}{38}=25.64\right)$ with a single-edge crack at $x_{c}=0.531$ ( $\left.X C=0.55\right)$. Ritz's result is shown for various crack ratios: $C R=0.0,0.13,0.5,0.8$

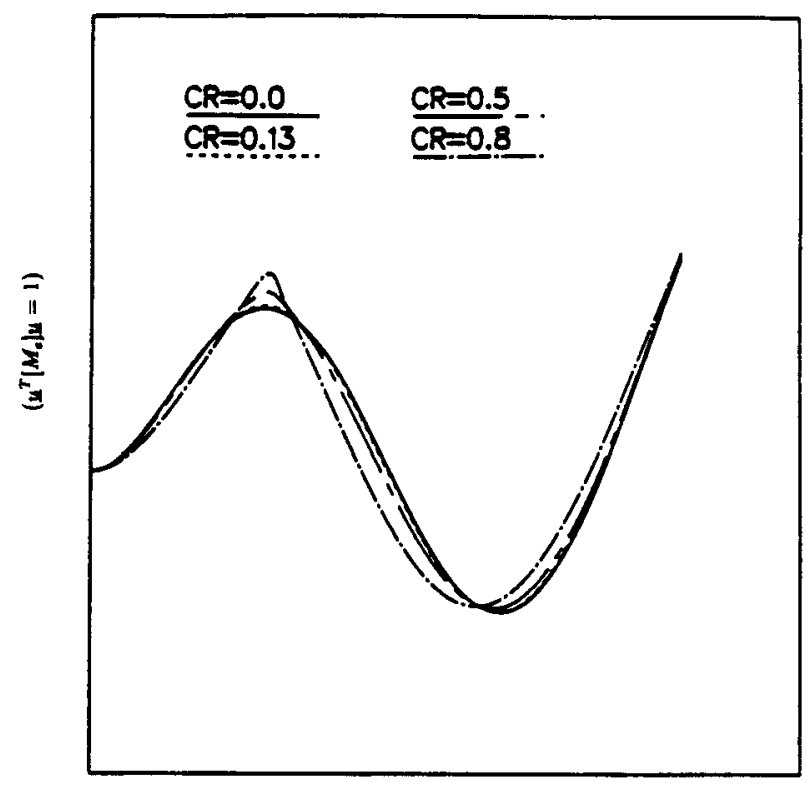

Figure 17. Third mode shape of a cantilevered beam $\left(\frac{1}{2 \mathrm{~g}}=25.64\right)$ with a single.edge crack at $x_{c}=0.31(X C=0.3)$. Ritz's result is shown for various crack ratios: $C R=0.0,0.13,0.5,0.8$.

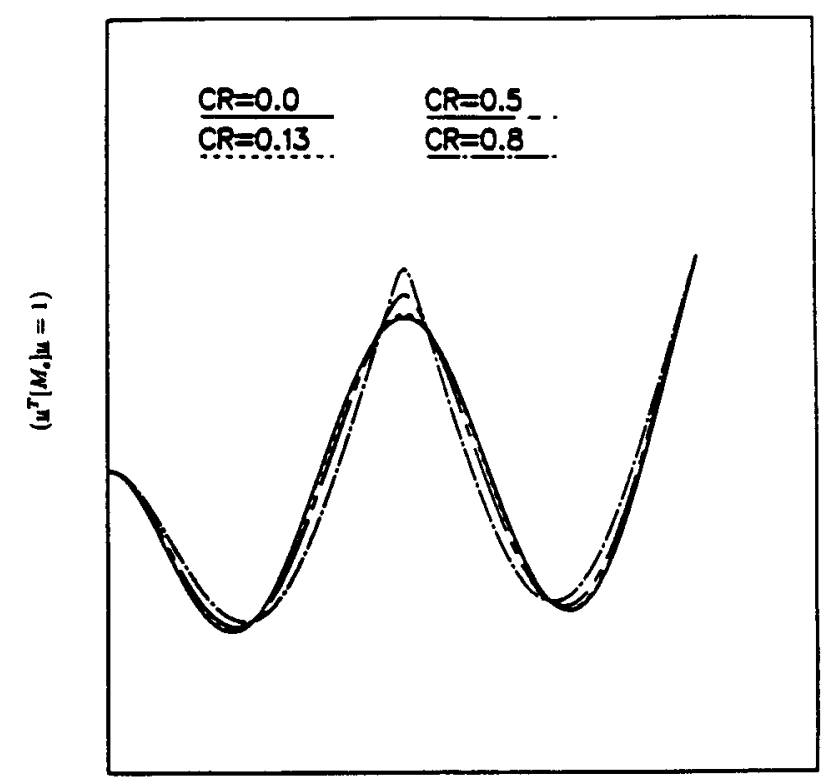

Figure 18. Fourth mode shape of a cantilevered beam $\left(\frac{t}{g}=25.64\right)$ with a single-edge crack at $x_{\epsilon}=0.51(X C=0.5)$. Ritz's result is shown for various crack tatios: $C R=0.0,0.13,0.5,0.8$ 


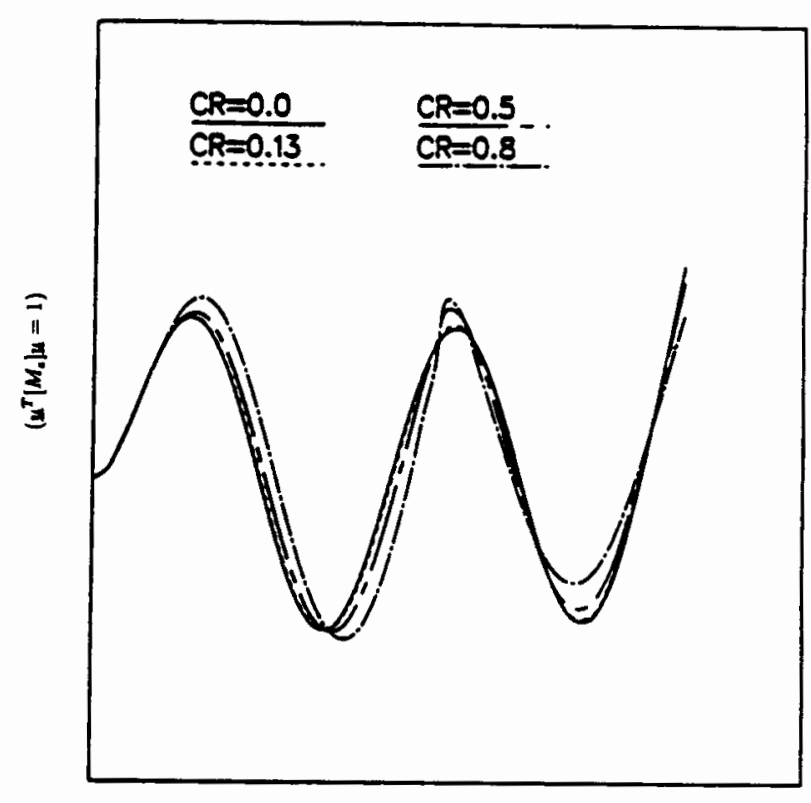

Figure 19. Fifth mode shape of a cantilevered bean $\left(\frac{1}{2 s}=25.64\right)$ with a single-edge crack at $x_{f}=0.7 i(X C=0.7)$. Ritz's tesult is shown for various crack ratios: $C R=0.0,0.13,0.5,0.8$

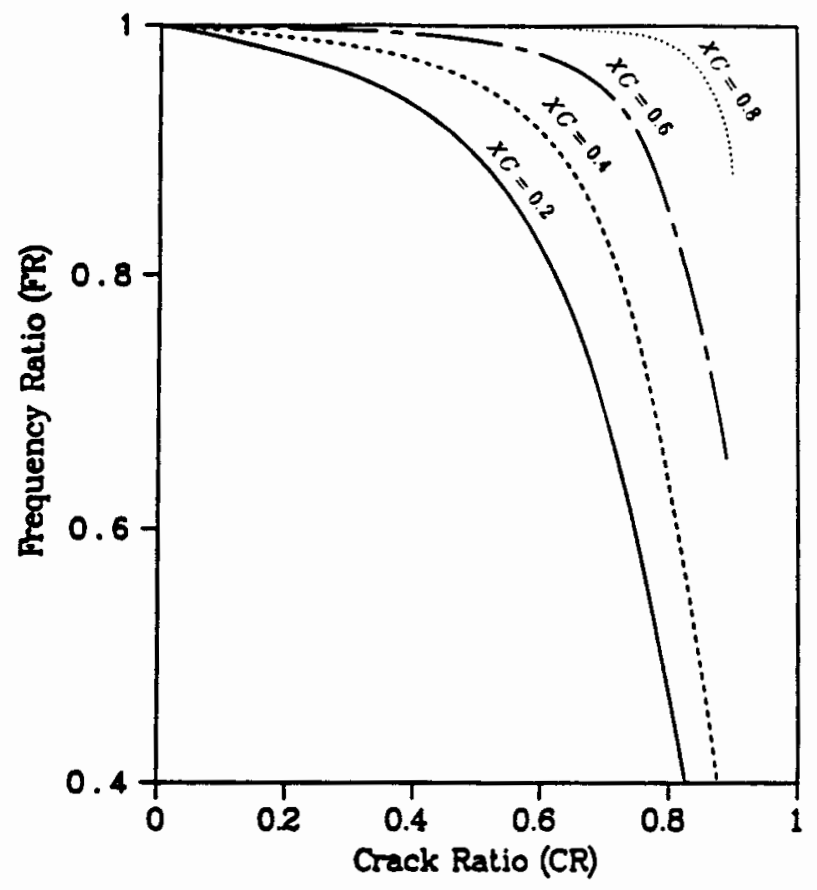

Figure 20. Fandamental gatural frequency in terms of crack depth for a cantilevered beam $\left(\frac{1}{2 z}=25.64\right)$. Ritz's result is shown for various erack positions: $. X C=0.2$, $0.4,0.6 .0 .8$.

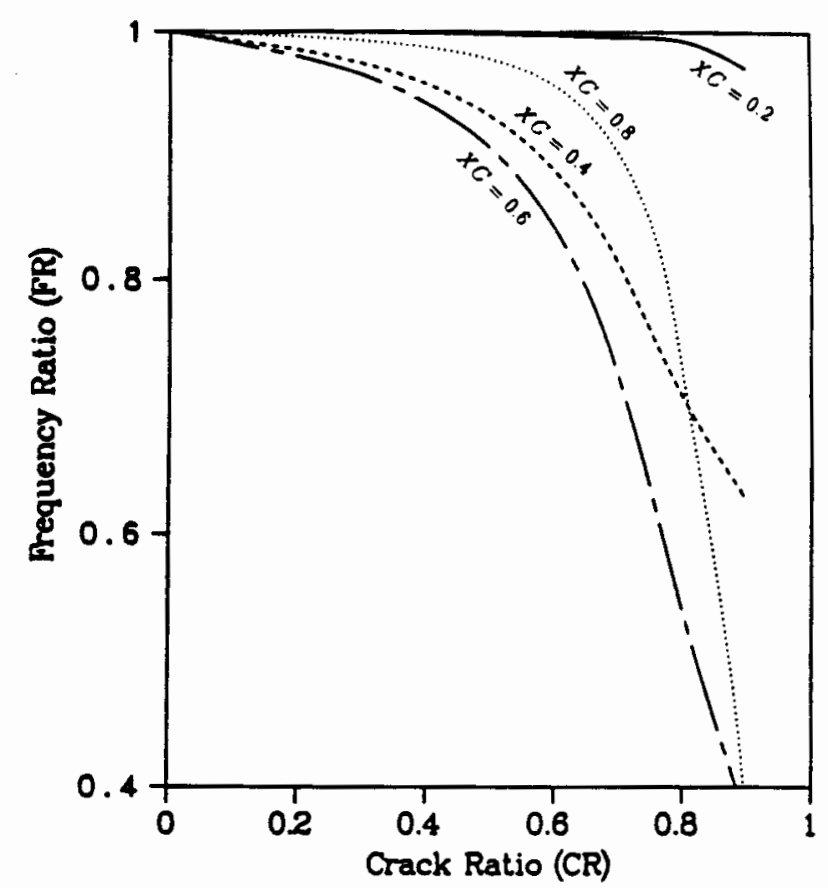

Figure 21. Second aatural frequency in terms of crack depth for a cantilevered beam $\left(\frac{1}{3 \mathrm{~d}}=25.64\right)$. Ritz's resuit is shown for various crack positions: $X C=0.2,0.4,0.6$.

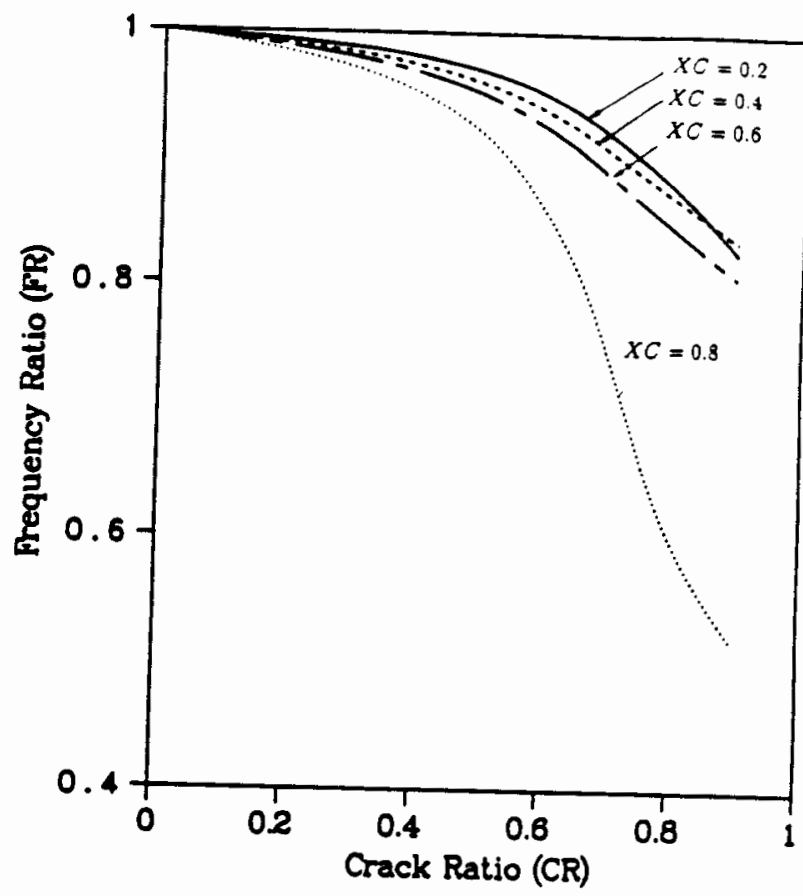

Figure 22. Third natural frequency in terms of crack depth for a cantilevered beam $\left(\frac{l}{28}=25.64\right)$. Ritz's result is shown for various ctack positions: $X C=0.2,0.4,0.6$. 


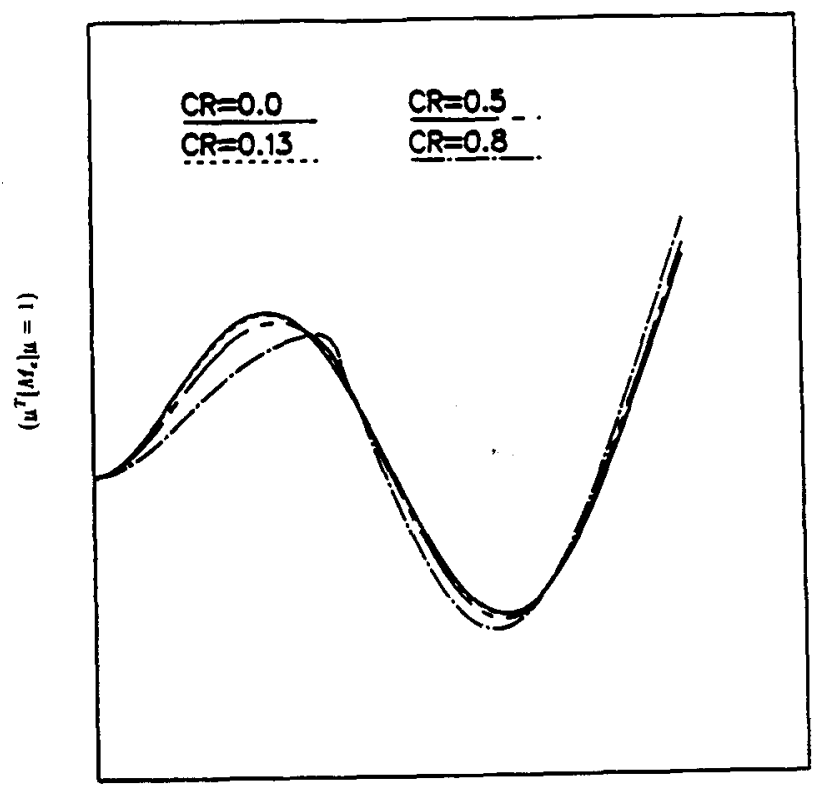

Figure 23. Third mode shape of a cantilevered beam ( $\left.\frac{1}{3 d}=25.64\right)$ with a single-edge crack at $x_{c}=0.4 l(X C=0.4)$. Ritz's result is shown for various crack ratios: $C R=0.0,0.13,0.5,0.8$

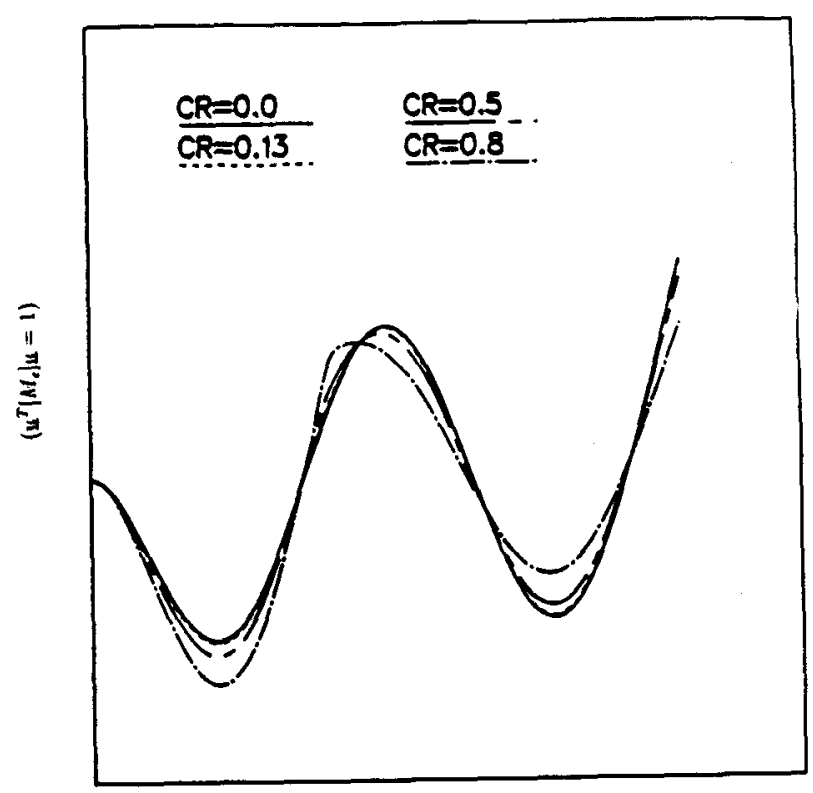

Table 1. Nanural frequeocy of uncracked and cracked beams (single crack)

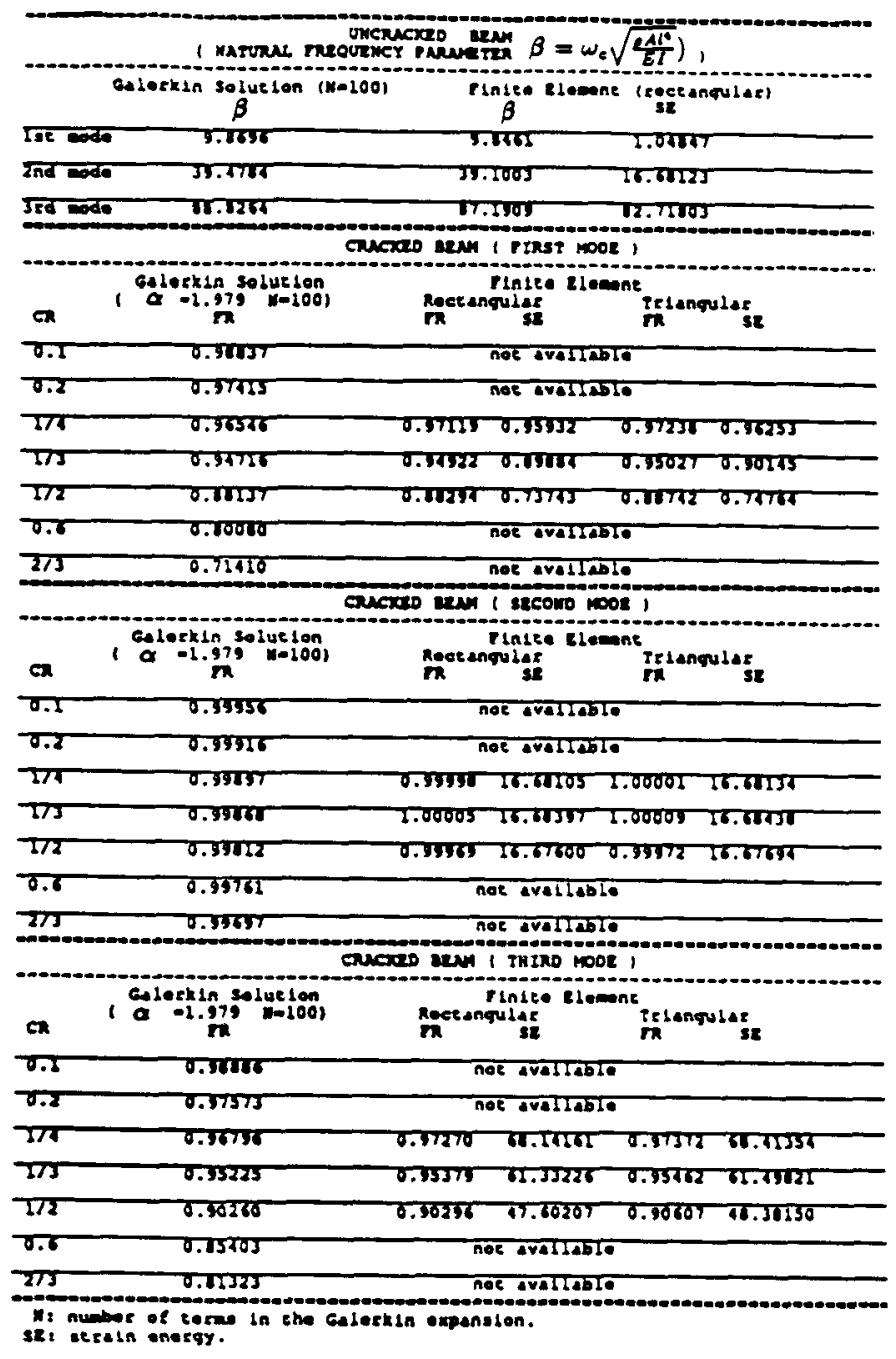

Figure 24. Fourth mode shape of a cantilevered beam $\left(\frac{1}{2 g}=25.64\right)$ with a single-edge crack 2t $z_{c}=0.4 l(X C=0.4)$. Ritz's result is shown for various ctack patios: $C R=0.0,0.13,0.5,0.8$. 